2 A Trypanosoma brucei ORFeome-based Gain-of-Function Library

3 reveals novel genes associated with melarsoprol resistance

4

5 Carter $\mathrm{M}^{1 \dagger}, \mathrm{Kim} \mathrm{HS}^{2 \dagger}$, Gomez $\mathrm{S}^{1}$, Gritz $\mathrm{S}^{1}$, Larson $\mathrm{S}^{1}$, Schulz $\mathrm{D}^{3 *}$, Hovel-Miner GA ${ }^{1 *}$

6

$7 \quad \dagger$ - Co-first

$8 \quad *$ - Co-corresponding

9

1) The George Washington University

Department of Microbiology, Immunology, and Tropical Medicine

2300 Eye St., Washington, D.C, 20037

ghovel_miner@gwu.edu

2) The Public Health Research Institute at the International Center for Public Health New Jersey Medical School - Rutgers, The State University of New Jersey 225 Warren Street, Newark, NJ 07103-3535

heesook@rutgers.edu

3) Harvey Mudd College

Department of Biology

1250 N. Dartmouth Ave. 


\section{LIST OF ACRONYMS AND ABBREVIATIONS}

25 ORF - Open reading frame

26 RIT - RNA-Interference Targeted

27 GOF - Gain-of-Function

28 RFU - Relative fluorescence units

29 DOX - doxycycline

$30 \mathrm{SM}$ - Single marker

31 LP - Landing pad

32 NGS - Next generation sequencing

33 GSH1 - $\gamma$-glutamylcysteine synthetase

34 TR - Trypanothione reductase

$35 \mathrm{~T}(\mathrm{SH})_{2}-$ Trypanothione

36 GSH2 - glutathione synthetase

37 ODC - Ornithine decarboxylase

38 TXN - Tryparedoxin

$39 \quad \mathrm{RR}$ - Ribonucleotide reductase

40

41

42

43

44

45 


\section{ABSTRACT}

48 Trypanosoma brucei is an early branching protozoan that causes Human and Animal

49 African Trypanosomiasis. Forward genetics approaches are powerful tools for

50 uncovering novel aspects of Trypanosomatid biology, pathogenesis, and therapeutic

51 approaches against trypanosomiasis. Here we have generated a T. brucei ORFeome

52 consisting of over $90 \%$ of the targeted genome and used it to make an inducible Gain-

53 of-Function library for broadly applicable forward genetic screening. Using a critical drug

54 of last resort, melarsoprol, we conducted a proof of principle genetic screen. Hits arising

55 from this screen support the significance of trypanothione, a key player in redox

56 metabolism, as a target of melarsoprol and implicate novel proteins of the flagellum and

57 mitochondria in drug resistance. This study has produced two powerful new genetic

58 tools for kinetoplastida research, which are expected to promote major advances in

59 kinetoplastida biology and therapeutic development in the years to come.

60

61

62

63

64

65

66

67

68

69 


\section{INTRODUCTION}

Trypanosomatids are a major parasitic lineage that include the African

72 trypanosomes, American trypanosomes, and Leishmania spp. (family

73 Trypanosomatidae, order Kinetoplastida), which collectively cause death and disease in

74 millions of people living in tropical and sub-tropical regions (1). There are no vaccines

75 against this family of parasites and the limited number of anti-Trypanosomatid drugs

76 present ongoing challenges of host toxicity, complex treatment regimens, and

77 burgeoning drug resistance (2). These evolutionarily ancient eukaryotes have highly divergent genomes from well-established model organisms with more than $50 \%$ of open reading frames (ORFs) annotated as hypothetical (3). Trypanosomatid parasites appear

80 to have diverged from a shared ancestor around 100 million years ago, yet have

81 retained a high proportion of orthologous gene clusters and chromosomal synteny. Of

82 the 9,068 genes in the Trypanosoma brucei (African trypanosome) genome, 6158 are

83 orthologous with both Trypanosoma cruzi (American trypanosome) and Leishmania

84 major (3). Many of the nonsyntenic regions of the genome appear to be directly

85 associated with organism specific aspects of parasitism, such as surface antigen

86 variation, leaving many biological functions highly conserved among kinetoplastida

87 parasites (3). Trypanosomatids share essential subcellular structures, such as the

88 flagellum, glycosomes (specialized organelles of glycolysis), and the kinetoplast (a

89 network of mitochondrial DNA). While reverse genetics based on well-established

90 models can promote discrete advances, forward genetics approaches have the potential

91 to uncover important aspects of kinetoplastida biology shared among orthologous

92 genes. 
T. brucei, the causative agent of Human African trypanosomiasis (HAT), has

94 traditionally been the most genetically tractable of the Trypanosomatid parasites.

95 Discoveries made in T. brucei have provided critical understanding to the biology of

96 other kinetoplastida as well as providing insights into eukaryotic evolution (4-7) and

97 uncovered broadly significant biological processes, such as GPI-anchors and

98 mitochondrial RNA-editing (8-10). For the past decade a whole-genome interfering-RNA

99 (RNAi) knock-down library has been the primary forward-genetics tool in T. brucei

100 resulting in identification of essential genes and genes associated with drug resistance

101 and pathogenesis, to name a few (11-13). A strength of the RNAi library and associated

102 RNA-Interference Targeted (RIT)-seq approaches is the identification of genes that

103 result in a loss-of-fitness phenotype (14). However, knockdown strategies may fail when

104 screening for a cellular event that requires a particular gene to be turned on rather than

105 turned off. Furthermore, they preclude identification of essential genes associated with

106 key processes (14). A Gain-of-Function library approach may be more effective in the

107 identification of genes whose expression is critical to the initiation of the event. For

108 example, Gain-of-Function screens have been employed to identify drug targets, as well

109 as resistance mechanisms (15-17), and in areas of basic biology have been critical for

110 discoveries in the areas of chromosome segregation and cell cycle, signal transduction,

111 transcriptional regulation, cell polarity, and stem cell biology (18). Thus, Gain-of-

112 Function screens are a powerful tool that can be used as a complement to loss-of-

113 function screening to uncover new biology in diverse areas.

Traditional methods of inducible gene library formation by cDNA synthesis and 
116 occurs post-transcriptionally, with 5' and 3'UTRs playing a major role in determining

117 steady state levels of their associated transcripts (19). Existing overexpression libraries

118 for T. brucei, generated by whole-genome fragmentation methods have demonstrated

119 the usefulness of this approach, for example, in the identification of the benzoxaborole

120 drug target (polyadenylation specificity factor, CPSF3), despite their somewhat limited

121 ability to control individual ORF expression (20-23). In addition, generation of an

122 ORFeome provides a tool that can be applied to the downstream generation of multiple

123 whole-genome methodologies including yeast 2-hybrid libraries, tagging libraries,

124 inducible expression libraries for Gain-of-Function studies, and complementation for

125 loss-of-function mutant phenotypes (24-27). A T. brucei ORFeome would permit

126 production of an inducible Gain-of-Function library, which is expected to be an ideal

127 design for the identification of novel gene functions based on observable phenotypes in

128 large scale screens.

129

African trypanosomiasis is transmitted by the tsetse vector and characterized by

130 a stage one bloodstream infection (though other tissues are implicated (28)) and a

131 stage two central nervous systems (CNS) infection, which is invariably fatal if left

132 untreated. HAT is predominantly caused by T. b. gambiense, whose stage two

133 infections can be treated by NECT (nifurtimox/eflornithine combination therapy) (2) and

134 fexinidazole (29). To a lesser extent HAT is caused by T. b. rhodesiense, which rapidly

135 progresses to stage two infection and host death. Melarsoprol, an arsenical compound, 136 remains the only drug available to treat stage two $T$. b. rhodesiense infections despite

137 treatment challenges, high levels of host toxicity, and increasing reports of drug

138 resistance (30). Following melarsoprol uptake by the parasite-specific P2 adenosine 
139 transporter (AT1 gene) and aquaglyceroporin transporter (AQP2 gene), intracellularly

140 metabolized melarsoprol interacts with trypanothione (30). Trypanothione is a

141 kinetoplastida specific form of glutathione required for thiol based redox metabolism

142 (31). While the inactivation of trypanothione by melarsoprol has been demonstrated,

143 and effects on cellular redox metabolism are known, it is has remained unclear if

144 melarsoprol has more than one intracellular target and if inactivation of trypanothione is 145 responsible for cell lysis (30).

146 In this study we sought to produce a whole-genome T. brucei ORFeome and use

147 it to create a Gain-of-Function library for genetic screens. Following PCR amplification

148 and cloning, the resulting ORFeome includes more than $90 \%$ of the targeted T. brucei

149 ORFs (>6500 of 7245 ORFs targeted). The ORFeome was then introduced into

150 bloodstream T. brucei (Lister427) to produce the T. brucei Gain-of-Function (GoF)

151 Library. Because of melarsoprol's clinical significance and unknown aspects of its

152 functionality, it was selected for a proof of principle GoF library genetic screen.

153 Following the isolation of melarsoprol survivor populations from the induced GoF library,

154 we identified 57 genes that were significantly overrepresented, which included an

155 anticipated biosynthetic precursor of trypanothione. In addition, we identified sets of

156 genes associated with gene expression, proteins predicted to localize to the

157 mitochondria, and others that localize to regions of the flagellum, whose association

158 with melarsoprol have not been reported previously. Collectively, these findings support

159 the usefulness of the broadly applicable T. brucei ORFeome developed herein, the

160 essential functionality of the resulting GoF library, and provide new insights into

161 mechanisms of melarsoprol-based cell killing and genes whose induced expression can 
162 promote drug tolerance. Thus, we have generated two genetic tools and a novel

163 dataset, which are all posed to provide new insights into Trypanosomatid biology,

164 pathogenesis, and aid in the development of novel therapeutics.

165

166

RESULTS

167 Generation of a Trypanosoma brucei ORFeome

T. brucei and others in the class kinetoplastida primarily regulate gene

169 expression post-transcriptionally based on transcript stability mediated by 5' and 3'

170 UTRs (19). Thus, previous production of overexpression libraries, based on whole-

171 genome fragmentation and cloning $(20,21)$, cannot ensure that individual ORFs are

172 similarly activated upon induction. To mitigate these problems, we produced a T. brucei

173 ORFeome using PCR amplification, which ensures the placement of each ORF under

174 the same inducible gene regulation system. ORF start and stop sites for all T. brucei

175 genes were obtained from available TREU927 ribosomal profiling data for 9200 genes

176 (32). The targeted T. brucei ORFeome filtered out 1956 ORFs that were unsuitable in

177 size (<100 bp or $>4500 \mathrm{bp}$ ), an undesired product (annotated as ribosomal genes,

178 VSGs, ESAGS, pseudogenes, or hypothetical unlikely), or if they were known multidrug

179 resistant channels (including MRPA whose overexpression is known to cause

180 melarsoprol resistance (33)). PCR primers for the resulting 7245 targeted ORFs were

181 designed in silico with matched melting temperatures and attB1 cloning sites were

182 added to both forward and reverse primers for subsequent Gateway cloning. The

183 resulting 7245 primer pairs for ORFeome amplification were synthesized and 
184

185

186

187

188

189

190

191

192

193

194

195

196

197

198

199

200

201

202

203

204

205

206

resuspended in 21 separate 384 -well plates that were organized by ORF size and gene annotations as either 'known' or 'hypothetical' (Table 1 and SUP. 1).

Each gene of the ORFeome was PCR amplified in 384-well format from

Lister427 genomic DNA. The general quality of the PCR reactions was assessed by the addition of SYBR green and measurement of the resulting Relative Fluorescence Units (RFU) (FIG. 1A). Based on the SYBR green assessment, the initial PCR reactions resulted in the successful amplification of $94 \%$ of the ORFeome (6820/7245 ORFs)

(FIG. 1B \& SUP. 2). Most PCR plates resulted in $\geq 90 \%$ amplification and only three of the 384-well plates between $80-90 \%$. To increase our coverage, we attempted to reamplify 429 failed PCR reactions and succeeded in producing 228 products resulting in a final total of 7039 PCR products amplified (97.2\% of the targeted genome).

The PCR reactions from each 384-well plate were pooled (10 ul from each well) into 21 corresponding PCR product pools, irrespective of the SYBR result, which maintained the product size range associated with each plate (Table 1). Each resulting size-sorted PCR pool was run on agarose gels and gel purified prior to gateway cloning (FIG. 1C). Thus, the DNA bands visualized on gels in Figure $1 \mathrm{C}$ represent hundreds of similarly sized PCR products arising from the same PCR plate, which were gel extracted prior to gateway cloning. Gateway cloning occurred in two steps: 1) introduction of ORFs into a general pENTR vector, which can function as a useful repository for the ORFeome, and 2) transfer of ORFs into a pDEST vector designed for ORF integration into the $T$. brucei rDNA spacer region under the rRNA promoter and tetracycline inducible gene regulation (FIG. 1D and SUP. 3 pSUN6 vector map). For the purpose of this study, we refer to the entire set of cloned ORFs as the pENTR 
207 ORFeome and the resulting set of pDEST (pSUN6 derived) libraries ready for

208 transfection into the T. brucei genome as the pTrypLib ORFeome.

\section{Sequencing, assessment, and final coverage of the T. brucei ORFeome}

The resulting $T$. brucei pENTR and pTrypLib ORFeome harboring plasmids were each pooled and prepared for Illumina sequencing by tagmentation. In order to assess which of the 7245 targeted ORFs were not present in the pENTR and pTrypLib

214 ORFeome harboring plasmids, we aligned the sequencing reads to the trypanosome 215 genome, removed PCR duplicates, and counted the number of reads corresponding to 216 each targeted gene. Because we knew that some of the targeted genes were highly 217 similar or duplicated, we aligned the reads under two modes, one that required unique 218 alignments and one that allowed multiple alignments. Both datasets were then 219 assessed to determine how many genes were 'missing' from each library; defined as 220 any targeted gene with zero aligned reads. The analysis of uniquely aligned reads 221 determined that 1,845 genes were missing from the pENTR library and 2,593 ORFs 222 were missing from the pTrypLib ORFeome. For reads that were multiply aligned, 1,656 223 ORFs were missing from the pENTR library and 2,420 ORFs were missing from the 224 pTrypLib ORFeome (Figure 2A - pENTR_1, pDEST_1). Our analysis indicated that many of the 'missing' ORFs were positive by SYBR 226 analysis following PCR and showed appropriately sized bands when randomly selected 227 missing ORFs were run on agarose gels (approx. 100 PCR products visualized, data 228 not shown). Thus, we predicted that our DNA input was exceeding the functionality of 229 the gateway system in these cloning stages and that the largest losses arose during the 
230 formation of the pENTR ORFeome. To address this limitation, PCR products

231 representing each missing ORF were isolated from the original PCR plates (using a

232 Perkin-Emer Janus Automated Workstation to select 2450 PCR products) and used to

233 produce 8 additional size-sorted ORF sub-pools (SUP. 4 - Table of cloning pools

234 including 'MISS_1-8'), which were gel purified and gateway cloned. These 8 new

235 plasmid pools were then combined with the previous pools and sequenced by

236 tagmentation, as described above.

237 Our final ORFeome analysis indicated that 457 and 292 ORFs were missing after

238 both rounds of cloning in the pENTR vector using unique or multiply aligned reads,

239 respectively. Gateway cloning into the final pDEST vector to form the pTrypLib

240 ORFeome resulted in 636 and 442 missing ORFs using unique or multiple alignments,

241 respectively (FIG. 2A - pDEST_Final and SUP. 5 - pENTR and pDEST missing

242 ORFs). Thus, the final pTrypLib ORFeome contains between 6,609 and 6,803

243 (depending on the type of analysis used) T. brucei ORFs, resulting in the successful

244 inclusion of $91-94 \%$ of the targeted ORFs.

245 The resulting sequencing data were used to assess both the overall coverage of

246 ORFs in the library and if specific ORF characteristics (length or annotation) resulted in

247 biases during ORFeome generation. First, we analyzed the coverage of each ORF in

248 the pTrypLib ORFeome. For ORFs that were present in the plasmid libraries (based on

249 reads aligned), we analyzed the count distribution in the sequenced pDEST plasmids

250 for both uniquely and multiply aligned reads. This analysis showed that most ORFs

251 resulted in $\log _{2}(\mathrm{RPKM})$ values between 0 and 10 (FIG. 2B and SUP. 6). Thus, the

252 number of poorly represented ORFs (RPKM < 1) is 195 for uniquely aligned reads and 
253369 for multiply aligned reads, representing $3 \%$ or $5 \%$ of all ORFs in the library,

254 respectively. The $\log _{2}(\mathrm{RPKM})$ value was then plotted against each corresponding ORF

255 length to determine if gene length affected their representation in the ORFeome (SUP.

256 6). No strong correlation was observed between ORF length and coverage in the

257 pTrypLib ORFeome, with a best fit line showing a small negative slope for both unique

258 and multiply aligned reads (-.000673 and -.000778 , respectively). Thus, in general,

259 shorter ORFs are not significantly more highly represented than longer ORFs.

260 Next, we sought to determine if there were patterns in the properties of genes

261 missing from the pTrypLib ORFeome. Histograms of ORF length were generated for

262 targeted genes, and compared to histograms of ORF lengths that were missing from the

263 pDEST library. The distribution of ORF lengths in the targeted library was similar to

264 those missing from the the pDEST library. These results indicated the successful

265 cloning of the ORFeome into pDEST and that the resulting libraries were not skewed

266 toward larger or smaller ORFs (FIG. 2C). Thus, we have generated both pENTR

267 ORFeome and pTrypLib ORFeome genetic tools that will broadly support progress in

268 Trypanosomatid research.

\section{Gain-of-Function library generation from the inducible pTrypLib ORFeome}

272 transfection into the genome using targeting to the rDNA spacer regions by homologous

273 recombination. To increase the frequency of transfection and ensure integration into a

274 single rDNA spacer we employed the established method of generating a Landing Pad

275 (LP) parental cell line that targets recombination to a single iteration of the rDNA spacer 
276 repeats. The stable LP cell line is then transfected with pRPa-Sce* harboring the IScel

277 enzyme and restriction site directed for recombination in the targeted rDNA spacer (FIG.

278 3A) (12). Thus, following IScel DNA double-stranded break induction by doxycycline

279 (Dox), transfected ORFs are recombined into the targeted rDNA spacer resulting in

280 more consistent transcription induction among library incorporated ORFs.

Validating inducible ORF transcription from a whole-ORFeome transfection

282 would be challenging to detect due to the overall complexity of the resulting cell

283 populations (harboring approx. 6,500 ORFs in multiple copies). Thus, prior to

284 transfecting the TrypLib ORFeome we sought to verify that the transcription of individual

285 ORFs within this library would be inducible and highly transcribed. Toward this goal we

286 generated a subset 'mini-library', in which one pDEST bacterial pool was plated (SUP.

287 2, Plate name - “2_Known”, ORF range 849-1056 bp) and six random individual

288 colonies isolated, plasmid DNA prepared, and sequenced to identify the ORF harbored

289 in each pDEST vector (FIG. 3B). The resulting plasmids were pooled equimolarly,

290 transfected into T. brucei, recovered as a single population of parasites (the ORFeome

291 'mini-library'), and then grown with or without Dox induction for 12 or 24 hours prior to

292 RNA extraction. The relative level of four ORFs were determined using RT-qPCR with a

293 forward oligo specific to pDEST (attB1site) and a reverse oligo specific to individual

294 ORF. Because these oligo pairs can amply the ORFs in the ORFeome library

295 (exogenous) and not endogenous ORF, we can measure the inducibility of our

296 ORFeome library. Levels of ORF transcripts were compared between uninduced and

297 Dox induced culture. All ORFs showed increased transcript levels following Dox

298 induction at 12 and 24 hours, 3 of the 4 ORFs resulted in approximately 10-30-fold 
299 increased after 12 hours and 50-600-fold increased after 24 hours (FIG. 3B). Thus, the

300 overall strategy of ORFeome exogenous transcription induction from pTrypLib cloned

301 ORFs is viable.

302 We then generated a doxycycline inducible T. brucei Gain-of-Function (GoF)

303 library from the pTrypLib ORFeome by transfection of 360 million cells (LP parent

304 following pRPa-Sce incorporation of IScel cassette) and selected in blasticidin. After 72

305 hours of selection mock transfected cells were dead and approx. 60 million cells

306 survived transfection (FIG. 3C - red line), which were then propagated to 3 billion cells

307 over 3 days to generate the T. brucei GoF library. Assessment of the resulting GoF

308 libraries by Illumina sequencing requires a specialized procedure to ensure that only the

309 introduced pTrypLib ORFs are sequenced and not the endogenous gene copies.

310 Illumina sequencing libraries are prepared using a gateway attB1 site specific forward

311 oligo, during PCR enrichment, and a similar custom sequencing primer complementary

312 to the attB1 site upstream of the introduced ORF. Thus, the resulting sequencing reads

313 primarily correspond to the 5 ' ends of the introduced ORF (SUP. 7A - Sequencing

314 strategy). Immediately following transfection and recovery in blasticidin, the T. brucei

315 GoF library consisted of 5,818 ORFs and then between 4,269 and 4,360 ORFs when

316 thawed and propagated for 3 days. The GoF 'INPUT' library, arising from 3 days of

317 library propagation, represents the full complement of ORFs present before a screening

318 condition is applied (FIG. 3D). It is unclear if the apparent loss of 1,459 genes arose

319 through an artifact associated with a relatively low number of NGS reads returned from

320 those samples or a true loss of content between library transfection and the subsequent

321 thawing of frozen library. 
Identification of overrepresented GoF library ORFs in a melarsoprol survivor

screen

325

Melarsoprol remains the only drug available for the treatment of second stage $T$.

b. rhodesiense infection, yet it is highly toxic and difficult to administer (30). In addition,

327 there have been increased reports of melarsoprol resistance and treatment failures in

328 sub-Saharan Africa since the 1990s, only some of which have been explained by

329 transporter mutations (34-37). Since melarsoprol has been widely studied, with both

known and unknown genes associated with its cytotoxicity and treatment failures, it was selected for a T. brucei GoF library proof of principle genetic screen.

To identify ORFs whose induced expression promote survival in the presence of

333 lethal doses of melarsoprol, we tested three concentrations of drug on the LP parental

334 cell line. Similar to previous reports, we observed that T. brucei LP cells died after 3

335 days in $35 \mathrm{nM}, 5$ days in $26 \mathrm{nM}$, and 7 days in $17 \mathrm{nM}$ melarsoprol (17 nM is

336 approximately two times the standard EC50) (FIG. 4A) (11). We predicted that the

337 progression of cell killing might be a critical factor in design of the GoF Library

338 melarsoprol resistance screen. That is cells that die too quickly might not have the

opportunity to successfully activate ORFs that confer resistance. In fact, when a genetic

340 screen was attempted in $35 \mathrm{nM}$ melarsoprol, the death of the culture was delayed by

341 one day in both uninduced and induced GoF library cells when compared with parental

342 landing pad, yet no survivor population emerged (FIG. 4B - Red dashed and dotted

343 lines overlap). In contrast, when cells harboring the GoF library were treated with $17 \mathrm{nM}$ 344 melarosoprol, uninduced cells (no Dox), took almost 4 days longer to die than the LP 
345

parental line (preliminary test, data not shown). Thus, a GoF forward-genetic screen was prepared in $17 \mathrm{nM}$ melarsoprol.

The experimental steps for the $17 \mathrm{nM}$ melarsoprol GoF genetic screen, including 24 hour pre-induction of all Dox treated cultures (Day -1), are shown at the bottom of Figure 4C. A freezer vial containing 100 million GoF library harboring cells was thawed and propagated for less than 3 days before splitting it into triplicate samples for the following conditions: GoF 'INPUT' (grown for 3 days without melarsoprol selection), GoF 17 nM No Dox (uninduced library maintained in melarsoprol), and GoF 17 nM +Dox (Dox induced library maintained in melarsoprol) (FIG. 4C). The parental cell line (LP) was maintained in melarsoprol alongside GoF library cultures and were below the limit of detection (FIG. 4 - grey dashed line at $1 \times 10^{2}$ cells $/ \mathrm{mL}$ ) on day 4 with complete cell death observed from day 5 until the completion of the screen on day 10. In contrast, uninduced (No Dox) GoF Library harboring cells showed partially delayed melarsoprol killing, with counts below detection at day 5 , but some observable surviving cells in culture until day 8 , after which all cells appeared dead (FIG. 4C - Blue dotted line). The melarsoprol treated, Dox induced GoF library was distinct from LP parent by day 3, and by day 5 populations of survivors emerged and grew similarly well in all three induced GoF (+Dox) biological replicates (FIG. 4C - Blue dashed line). At day 7 the biological replicates of induced (+Dox) GoF library melarsoprol survivor cultures were split into media with and without Dox during continued treatment with 17nM melarsoprol (FIG. 4C - Green lines). The uninduced (no Dox) cultures died rapidly over the next 2 days (dotted green line), whereas the induced GoF library, once again, survived and grew robustly (dashed green line) in the presence of melarsoprol. Thus, survival in the 
presence of melarsoprol was dependent on GoF library induction. Biological triplicate cultures of melarsoprol survivors were harvested from both GoF 'MEL-1x' and GoF 'MEL-2x' (resulting from the day 7 split) on day 9 and day 10 respectively (FIG. 4C blue and green dashed lines, red circles indicate the date of harvest). Genomic DNA

372 from biological triplicate cultures of untreated INPUT (day 3, black circle), GoF 'MEL-1x',

373 and GoF 'MEL-2x' cultures (9 cultures total grown to 1 million cells per $\mathrm{mL} 200 \mathrm{~mL}$ each) was prepared for NGS analysis.

To identify the pTrypLib ORF incorporated into the GoF library harboring cells recovered from each replicate and condition (INPUT, GoF MEL-1x, and GoF MEL-2x),

377 genomic DNA was fragmented and prepared for ORFeome specific Illumina sequencing 378 (shown in SUP. 6). During PCR enrichment INPUT samples were prepared in two ways: 379 long elongation time (INPUT1, 75 seconds) and shorter elongation time (INPUT2, 20 380 seconds). Sequencing data was obtained in biological triplicate from the two sets of 381 INPUT libraries (no melarsoprol treatment) and the two sets of melarsoprol selected 382 parasites (MEL-1x and MEL-2x). We performed principle component analysis on the 383 sequencing data from both unique and multiple alignment datasets (FIG. 4D). The PCA 384 analysis shows two clearly separated clusters for untreated and melarsoprol treated 385 samples, with most biological replicates clustering proximal to one another. Melarsoprol 386 treated samples were distinct from INPUT and show more variation between samples 387 (FIG. 1D). In order to check whether ORF length influenced the level of coverage in the 388 genomic INPUT libraries, we analyzed whether ORF representation in the INPUT

389 libraries correlated with ORF length. We observed, at best, a weak negative association 390 between gene length and normalized read count (slopes of -.00042 and -.00045 for 
391 unique and multiple alignment analysis, respectively), indicating that ORF

392 representation in the library is largely independent of ORF length (SUP. 8)

We reasoned that any gene whose induction contributed to melarsoprol

394 resistance should be overrepresented in libraries generated from melarsoprol survivor

395 populations. The designation 'overrepresented' indicates that the normalized read count

396 for a particular ORF in the presence of melarsoprol is higher in libraries arising from

397 doxycycline induced parasites harboring the GoF library (MEL-1x and MEL-2x) when

398 compared with the untreated GoF library (INPUT), which suggests that overrepresented

399 genes have enhanced survival in the presence of drug. To determine the fold change

400 that represents a valid difference between melarsoprol treated and untreated conditions,

401 we compared each of the three biological replicates of INPUT2 to one another and

402 counted the number of ORFs with a 1.5, 2.0, or 4.0-fold in normalized read count (FIG.

403 5A - INPUT2 replicates compared pairwise). By evaluating the biological variation

404 between similarly treated replicates we found that while many ORFs varied in

405 normalized read count by greater than 1.5-fold between replicates (more than 300 ), very

406 few ORFs varied by greater than 4-fold (FIG. 5A, similar results obtained from INPUT 1,

407 data not shown). Thus, we used a 4-fold change in normalized read count between

408 melarsoprol treated and untreated samples as the minimum threshold for identifying an

409 ORF as overrepresented (a 'hit') in this study.

410 In order to identify ORFs overrepresented in melarsoprol survivor populations,

411 we aligned reads from each of the 12 samples (3 reps of each: INPUT1, INPUT2, MEL-

$4121 \mathrm{x}$, and MEL-2x) to the genome, and then calculated the number of reads that fell within

413 the first 100bp of each of the ORFeome targeted ORFs (SUP. 7B - Example read 
414 alignments). DESeq was used to normalize read counts between libraries (38). We

415 performed this analysis for uniquely aligned reads and multiple alignments. DESeq was

416 used to identify genes that were 'differentially expressed' between melarsoprol selected

417 samples and INPUT samples with a p adjusted value of $<0.05$ (FIG. 5B) (38). In this

418 context, 'differential expression' simply refers to an ORF being overrepresented or

419 underrepresented in the melarsoprol selected samples. To be considered as a potential

420 hit, we required a minimum normalized read count of 5 in INPUT samples to avoid

421 ORFs being called as overrepresented that were simply poorly covered. To identify

422 putative hits, we then asked which of the differentially represented ORFs with and $p$

423 adjusted value $<0.05$ had a normalized read count $>4$-fold higher in 3 biological

424 replicates of melarsoprol treated populations (MEL-1x and MEL-2x) compared to

425 untreated populations (INPUT1 and INPUT2). Four different comparisons were

426 analyzed using this pipeline: INPUT1 vs. Mel-1x, INPUT1 vs. Mel-2x, INPUT2 vs. Mel-

427 1x, and INPUT2 vs. Mel-2x (FIG. 5B). Figure 5C shows a volcano plot of DESeq

428 generated significance values compared with fold change for the comparison between

429 INPUT2 and MEL-1x. After hits had been called for each individual comparison, we

430 identified the hits common between all 4 comparisons for both uniquely and multiply

431 aligned reads (FIG. 5D and SUP. 9 - Tables of all comparisons). These analyses

432 resulted in the identification of 57 overrepresented ORFs (uniquely aligned) in the GoF

433 melarsoprol survivor populations compared to INPUT populations. In the comparison of

434 INPUT2 vs. MEL-1x depicted in a volcano plot, we observe that these 57 ORFs

435 common to all the comparisons were among the most highly overrepresented genes

436 and with some of the lowest $P$ adjusted values determined by DESeq (FIG. 5C - blue 
437 dots). Similar results were obtained for all comparisons between melarsoprol selected

438 and input samples. We conclude that our analysis is reasonably robust at identifying the

439 most consistent, statistically significant, highly overrepresented genes in melarsoprol

440 treated samples compared to untreated samples.

$441 \quad$ The 57 genes overrepresented in melarsoprol survivor populations consist of 25

442 genes annotated as conserved hypothetical and among the genes with annotations

443 most have only putative functional assignments (SUP. 10). Among the top 5

444 overrepresented hits we identified that the gene encoding $\gamma$-glutamylcysteine synthetase

445 (GSH1, Tb927.10.12370) was 191-fold higher among melarsoprol survivors when

446 compared with input control. GSH1 is required for the biosynthesis of trypanothione, a

447 known target of melarsoprol, and its overexpression has been demonstrated to promote

448 melarsoprol resistance in laboratory studies (33). This finding supports the role of

449 trypanothione as one of the major intracellular targets of melarsoprol and the usefulness

450 of the GoF library in the direct identification of drug targets. Using available functional

451 annotations (tritrypdb.org), we found that 15 of the 57 overrepresented hits are either

452 known or implicated in gene expression regulation. It is not clear at this time if this is a

453 true aspect of melarsoprol functionality or whether this is an artifact arising from the

454 nature of the GoF library; namely increased transcript stability and translation of diverse

455 proteins having a nonspecific effect on cell viability in the presence of drug. Only

456 through further GoF library validation will we know if gene expression is truly associated

457 with melarsoprol resistance. We then utilized publicly available resources (tritrypdb.org

458 and tryptag.org) to develop additional categories based on subcellular localization (from

459 proteomic and microscopic studies) and published protein functions. We found that the 
460 majority of overrepresented genes encoded proteins that localize to the mitochondria

461 and kinetoplast (10 genes, shown in Table 2) or the flagellum (9 genes, shown in Table

462 3) (SUP. 10 - Hits listed with categories). The prevalence of mitochondrial and flagellar

463 proteins arising from the melarsoprol GoF screening may implicate previously

464 unrecognized melarsoprol drug targets or pathways of drug tolerance.

Overexpression of genes identified in melarsoprol GoF screen result in increased

\section{7 drug tolerance}

GoF library induction in the presence of melarsoprol resulted in the isolation of survivor populations harboring a consistent set of overrepresented genes. The growth environment arising from the induced expression of many genes is likely distinct from

471 cell populations overexpressing only one gene and may influence population survival

472 through the secretion of unknown factors or other events. To determine if the

473 overexpression of individual genes identified among the 57 overrepresented hits can

474 contribute to melarsoprol resistance, we cloned a subset of genes in to the pLEW100v5-

475 BSD vector, which permits Dox inducible gene expression in a manner similar to the

476 GoF library itself. Bloodstream form T. brucei (SM) were transfected with linearized

477 plasmids harboring individual genes for overexpression and, following their recovery

478 were, assayed for their effects on melarsoprol sensitivity by alamarBlue assays. The

479 gene encoding $\gamma$-glutamylcysteine synthetase (Tb927.10.12370, GCS1), which is

480 responsible for the biosynthesis of glutathione used to form trypanothione, was among

481 the most overrepresented genes (190-fold), is essential, and its overexpression has

482 been linked to resistance in previous studies $(39,40)$. As anticipated induced 
483

484

485

486

487

488

489

490

491

492

493

494

495

496

497

498

499

500

501

502

503

504

505

overexpression of GSH1 resulted in an increase in the EC50 of melarsoprol of almost 2fold (FIG. $6 \mathrm{~A}-\mathrm{SM}=\mathrm{EC50} 7.5 \mathrm{nM}$ and OE GSH1 $=14.5 \mathrm{nM})$ suggesting that increased expression of components of this pathway can produce more trypanothione and diminish the efficacy of melarsoprol. The trypanothione pathway (FIG. 6B) and its implications for melarsoprol resistance are covered in more detail in the discussion. We also found that the overexpression of the hypothetical conserved gene Tb927.7.2780 (overrep. 322-fold) resulted in a 2-fold shift in EC50 (FIG. 6C - OE = EC50 15.1). The protein encoded by Tb927.7.2780 is a predicted post-transcriptional activator associated with mRNA stability or increased translation (41) and is annotated as an essential gene based on RIT-seq analysis (42). Whether melarsoprol resistance associated with $T b 927.7 .2780$ arises from direct effects on gene expression of known pathways, such as trypanothione biosynthesis enzymes, or more general effects on global gene regulation remains to be determined. A similar shift in EC50 was also observed upon overexpression of Tb927.9.15020 (FIG. 6C - OE = EC50 14.0), which encodes a hypothetical protein of unknown function (overrep. 30.1-fold) that we categorized as flagellar (axonemal) based on TrypTag.org localization data (images shared ahead of online deposition). It is unclear at this time how flagellar proteins may participate in melarsoprol resistance, discussed in more detail below. Multiple genes encoding mitochondrially localized proteins were cloned for similar overexpression analyses, but resulted in assay challenges arising from either growth defects, inability to reduce alamarBlue, or both. Because cell viability in alamarBlue assays is reported based on the reduction of the active reagent (resazurin) by mitochondrial redox metabolism, it is not a surprise that the effects mitochondrial proteins could not be 
assayed in this manner and may indicate their association with the trypanothione redox

507

508

509

510

511

512

\section{DISCUSSION}

514

515

516

517

518

519

520

521

522 genetics and unbiased studies based on the T. brucei genome (TREU927): an

523 ORFeome consisting of over 6500 ORFs and an inducible Gain-of-Function library

524 harbored in T. brucei parasites (Lister427) for genetic screening. To validate the

525 functionality of the T. brucei GoF library, we conducted a genetic screen using the

526 troublesome drug melarsoprol and identified both an anticipated target as well as novel

527 genes that may reveal new facets for melarsoprol's mechanism of action. 
Melarsoprol has a complex history dating back to early uses of arsenic treatment

529 of HAT, treatments which are of specific significance because of their ability to cross the

530 blood-brain barrier and treat second stage infections (30). In vivo melarsoprol is rapidly

531 metabolized to other trypanocidal metabolites, including melarsen oxide, which interacts

532 with trypanothione to from MelT, a potent inhibitor of trypanothione reductase (TR) (11).

533 Yet, the precise mechanism of melarsoprol cell killing has remained unclear and the

534 complex forms of metabolized melarsoprol may have additional intracellular targets.

535 Melarsoprol drug resistance and treatment failures have been on the rise since the

536 1990s and while most resistance has been connected to mutations in aquaglyceroporin

537 transporters, numerous treatment failures remain unexplained (30). The trypanothione

538 biosynthetic precursor, $\gamma$-glutamylcysteine synthetase (GCS1), was among the most

539 overrepresented genes in melarsoprol survivors and is known to result in melarsoprol

540 resistance upon laboratory overexpression $(39,40)$. Upon deeper excavation of the

541 melarsoprol GoF screen data, we observed that a number of genes upstream and

542 downstream of $\mathrm{T}(\mathrm{SH})_{2}$ with enriched reads in melarsoprol survivors, but did not meet

543 the criteria to be called overrepresented (SUP. 11). For instance, both of the T(SH)2

544 biosynthetic precursors ornithine decarboxylase (ODC, Tb927.11.13730) and

545 glutathione synthetase (GSH2, Tb927.7.4000) were partially overrepresented in some

546 cultures of melarsoprol survivors (but not all). Downstream from $\mathrm{T}(\mathrm{SH})_{2}$, we saw

547 increased levels of trypanothione reductase (TR, Tb927.10.10390), a trypanothione

548 dependent peroxidase (Tb927.7.1140), and ribonucleotide reductase (RR,

549 Tb927.11.7840), in some survivor cultures. The generation of dNTPs by the action of

550 RR has known effects on kDNA replication (43) and melarsoprol treatment was recently 
551 shown to stall cell cycle (44), which might suggest inhibition of DNA synthesis as a

552 mechanism of cell killing. Collectively these findings may further support the

553 trypanothione pathway as a primary target of melarsoprol-based cell killing.

554 Among the 57 significantly overrepresented genes approximately $45 \%$ are

555 annotated as hypothetical. The majority of overrepresented genes with functional

556 predictions are associated with gene expression (15 genes) with the largest groups

557 associated post-transcriptional regulation (6 genes) or splicing factors (5 genes).

558 Notably, one of the overrepresented genes associated with post-transcriptional gene

559 expression regulation (Tb927.10.1490, MKT1L) was also a high significance gene in

560 melarsoprol RNAi screening (45). For overrepresented genes without functional

561 annotations we focused on the cellular localization of the encoded proteins (tritrypdb.org

562 and tryptag.org) and identified a preponderance of proteins that localize to either the

563 mitochondria (11 genes) or flagellum (9 genes) (FIG. 7). The flagellar proteins have

564 diverse localizations in procyclic form (insect stage) parasites, that include localization

565 to the flagellar pocket and along the body of the organelle (axonemal and paraflagellar

566 rod, specifically). Since the melarsoprol transporter AQP2 is specifically localized to the

567 flagellar pocket in bloodstream form parasites (46), one could speculate that

568 overexpression of a protein that interferes with drug transport in this region might

569 enhance resistance. Two of the overrepresented flagellar genes, one axonemal

570 ( $T b 927.5 .4150)$ and one localized to the hook complex (Tb927.1.1020), were

571 associated with a loss of fitness in RIT-seq screens, which is indicative of their

572 essentiality. It will be the subject of future studies to determine how flagellar proteins

573 might contribute to drug resistance or if they are viable drug targets. 
Connections between $\mathrm{T}(\mathrm{SH})_{2}$ and mitochondrial redox reactions have been

575

576

577

578

579

580

581

582

\section{3}

584

585

586

587

588

589

590

591

592

593

594

595

596

widely established (31) and the anti-Trypanosomatid drugs pentamidine and nifurtimox have been linked to specific mitochondrial functions $(44,47)$. Yet, the data arising from the GoF screen described herein represent the first genetic links between melarsoprol and the mitochondria. Furthermore, since the majority of the GoF proteins that localize to the mitochondria are hypothetical or have only putative functionality, the 11 mitochondrial genes identified here have the potential to uncover novel biology in the mitochondria and kinetoplast. Among them, the best studied gene encodes $\beta$-ketoacylACP reductase (Tb927.2.5210, 23-fold overrep.), a key member of the Type II Fatty Acid Biosynthesis (FAS) pathway in the mitochondria. Interestingly, lipoic acid, whose biosynthesis arises from Type II FAS, is an alternative redox active low molecular mass thiol alternative to $\mathrm{T}(\mathrm{SH})_{2}(31,48)$. Further studies will be required to determine if overexpression of $\beta$-ketoacyl-ACP reductase and other novel mitochondrial proteins promote melarsoprol resistance either through their redox potential or other functions in the mitochondria.

The functionality of the T. brucei ORFeome could be extended to generate additional genetic tools, such as, yeast two-hybrid libraries, tagging libraries, and in dominant negative genetic screening approaches $(22,26,49,50)$. Based on the conservation of orthologous gene clusters among kinetoplastida (3), we also predict the ORFeome could be used in other Trypanosomatids to generate orthologous Gain-ofFunction libraries and other tools. The vast majority of genes overrepresented in melarsoprol survivor populations ( $80 \%$, see SUP. 13) are conserved among sequenced kinetoplastida genomes. This supports the use of these tools to broadly 
597 expand our understanding of gene functions in this family of parasites. Notably,

598 approximately $25 \%$ of all genes overrepresented in melarsoprol survivors were

599 identified as resulting in loss-of-fitness in RIT-seq screening (26-23\% depending on life

600 cycle stage) (SUP. 12 - RIT-seq compared), which validates the usefulness of the GoF

601 library tool in identifying essential genes, an acknowledged limitation of RNAi based

602 screening (14,51). Additionally, we found genes overrepresented in the melarsoprol

603 GoF screen that were also identified in nifurtimox and melarsoprol RNAi based screens

604 (SUP. 12 - RIT-seq compared) (51). Thus, we see the GoF library as a powerful new

605 tool, complementary to RNAi knock-down approaches, to expand our understanding of

606 drug targets and pathways of resistance. In future drug resistance screens, it will be

607 useful to determine if certain sets of genes are commonly overrepresented in survivor

608 populations and contrast that with genes that appear uniquely associated with an

609 individual treatment. The tools and discoveries arising from this study are expected to

610 support broad advances in basic biology, pathogenesis, pathways of drug resistance,

611 and the discovery of new therapeutic targets in Trypanosomatid parasites. 


\section{MATERIALS AND METHODS}

\section{Oligo design and PCR amplification of the ORFeome}

In order to design oligos for the ORFeome, we used gene annotations generated

623 from ribosome profiling data (32). We first filtered out genes that were either $<100 \mathrm{bp}$ in

624 size or $>4,500 \mathrm{bp}$ in size. We then filtered out any genes annotated as pseudogenes,

625 hypothetical protein unlikely, VSG, ISG, ESAG, GRESAG, or ribosomal protein.

626 Following this procedure, we used custom python scripts to design forward and reverse

627 primers. If the first $30 \mathrm{bp}$ of the ORF had a Tm $>55$, this primer was used. Otherwise, a

628 nucleotide was added one at a time until the primer had a Tm of greater than 55 . A

629 reverse primer was designed using the same procedure for the reverse complement of

630 the last 30 nucleotides. Many genes in the $T$. brucei genome have identical beginnings

631 and ends, and we did not wish to order duplicate sets of primers that were identical to

632 each other. For this reason, only one primer pair was kept for duplicated or highly

633 similar genes, and a record was made of any pair that was eliminated based on

634 duplication. All excluded ORFs are listed in SUP. 14. Finally, the ATT cloning

635 sequences 5'-GGGGACAAGTTTGTACAAAAAAGCAGGCT and 5'-

636 GGGGACCACTTTGTACAAGAAAGCTGGGT were added to the forward and reverse

637 primers, respectively.

Each 384-well plate containing mixed forward and reverse primer pairs were

639 diluted to $10 \mathrm{mM}$ in primer dilution plates. PCR plates were filled with a master mix for

640 KOD Hotstart (Novagen \#71086) PCR reactions containing Lister427 gDNA according

641 to manufacturer's specifications and primer pairs specific to each ORF were transferred

642 into the associated well number for each plate by TECAN Freedom EVO 150 pipetting 
643 instrument. PCR reaction conditions followed manufacturers specifications for $25 \mu \mathrm{L}$

644 reactions with annealing temperature of $55^{\circ} \mathrm{C}$ and elongation times ranging from $10-60$

645 seconds depending on the anticipated lengths of products in each plate. Following PCR

646 reaction completion SYBR Green I (Invitrogen S-7563) was added to each well at 1:10

647 PCR volume for a final concentration of 10x SYBR and relative fluorescence units

648 (RFU) were measured on a BioTek Synergy H4 Hybrid Multi-Mode Microplate reader in

649 comparison to a standard dilution of known DNA concentration. The resulting PCR

650 reactions from all wells of each 384-well plate were then pooled prior to agarose gel

651 separation and DNA extraction. PCR reactions called 'negative' based on SYBR (less

652 than 10,000 RFU) were revisited by isolating primer pairs from original 384-well plates,

653 using a Perkin-Emer Janus Automated Workstation, into two new PCR plates (SUP. 2 -

654 NEG_PICKS_\#1 and NEG_PICKS_\#2), which were then amplified using KOD Hotstart

655 specifications and extracted from agarose gels as described. The resulting extracted

656 DNAs from each PCR product pool were utilized in subsequent gateway cloning

657 reactions.

658

\section{Gateway cloning and plasmids}

The pENTR library was generated by cloning each size-sorted PCR product pool

661 into pENTR 221 Gateway Entry vector according to manufacturer's specifications

662 (https://www.thermofisher.com/us/en/home/life-science/cloning/gateway-cloning.html)

663 and transformed into One Shot $c c d B$ Survival Competent Cells by electroporation. The

664 resulting transformants were plated on large LB plates, assessed for efficiency of

665 transformation, and bacterial colonies isolated from plates into LB liquid, which was split 
666 for maxi preps of plasmid and storage at $-80^{\circ} \mathrm{C}$ in glycerol stocks. A T. brucei specific

667 pDEST vector, pSUN6 (map shown in SUP. 3) was generated by introducing a $c c d B$

668 gateway cassette into a pLEW type vector (52) for incorporation into the T. brucei

669 genome based on rDNA spacer homology, blasticidin selection, and ORF transcription

670 from an rDNA spacer promoter repressed by two tetracycline operators. Pools of

671 pENTR plasmids harboring size-sorted ORF populations were combined with pSUN6 in

672 LR clonase reactions and transfected into ccdB Survival Competent Cells following

673 manufacturers guidelines as described. The resulting transformants were plated on

674 large LB plates, assessed for efficiency of transformation, and bacteria and DNA

675 isolated as described for pENTR steps above. The resulting extracted DNAs from both

676 pENTR and pDEST ORFeome gateway cloning steps were assessed by NGS,

677 described below. Following the initial assessment of 'missing' ORFs from both pENTR

678 and pDEST cloning reactions, 'missing' PCR products were isolated from original

679 plates, using a Perkin-Emer Janus Automated Workstation, to generate 8 new pools of

680 size sorted PCR reactions (SUP. 4), which underwent the same series of pENTR and

681 pDEST (pSUN6) cloning reactions described above and subjected to NGS analysis.

682 The final set of ORFeome NGS validated (below) pDEST vectors are pooled to

683 generate a single pTrypLib ORFeome for introduction into the T. brucei genome.

\section{T. brucei cell lines and transfections}

Cell lines were generated from Lister427 bloodstream-form trypanosomes

687 derived from the 'single marker' (SM) line (53) and maintained in HMI-9 medium (54) 
689 generated using plasmids gifted to us by the Alsford Lab and validated for inducible

690 gene expression, prior to transfection with pRPaSce* as described $(12,51)$. LP parasites

691 harboring the I-Scel restriction site and enzyme in the targeted rDNA spacer are

692 doxycycline induced to permit IScel cutting prior to pTrypLib ORFeome transfection by

693 AMAXA Nucleofector (55). To generate the T. brucei GoF library described here, four

$694100 \mathrm{ml}$ flasks grown to $\sim 1$ million cells/mL were AMAXA transfected in four separate

695 transfection reactions, which were then pooled into a single cell population in $500 \mathrm{~mL}$ of

696 HMI-9 containing blasticidin and recovered in a large roller flask (FIG. 3C). An additional

697 four transfections were completed in parallel with TE (Mock) to compare outgrowth with

698 GoF Library transfection. The resulting blasticidin recovered GoF Library population was

699 expanded to an $800 \mathrm{~mL}$ culture at $\sim 1$ million cells per $\mathrm{mL}$ and saved in aliquots of 100

700 million cells per vial for future genetic screens. Cells were also sampled prior to freezing

701 for NSG analysis ('GoF library', described below) and after freeze thaw (INPUT1).

$702 \quad$ Single gene overexpression cell lines were generated by cloning ORFs of

703 interest into pLEW100v5-BSD (https://www.addgene.org/27658/), which following

704 validation were digested with Notl and transfected into SM by AMAXA.

705

706 Quantitative PCR assessment of individual ORF induction

707 Individual cloned ORFs were selected randomly from pDEST colonies plated on

708 LB originating from the pool '2_known', ORFs confirmed by traditional DNA sequencing,

709 and DNAs arising from 6 individual ORF harboring pDEST vectors were transfected into

710 LP harboring pRPaSce* by AMAXA as described above. This generated a 'Mini-Library'

711 following transfection and recovery, which was split into No Dox and +Dox conditions for 
71224 hours, RNA extracted, and cDNA prepared by Superscript III (ThermoFisher

713 \#18080044), prior to qPCR analysis. Quantitative PCR data was produced on a Bio-Rad

714 CFX96 Real-Time PCR Detection System with iTaq Universal SYBR Green Supermix

715 (Bio-Rad, \#1725121) reagent using a forward primer unique to cloned ORFeome genes

716 (5'-GGGGACAAGTTTGTACAAAAAAGCAGGCT) and reverse primer to each gene

717 analyzed: Tb927.8.2230 (PRIMER: 5'- CACGGTTTTTGCCCATTCGT), Tb927.1.4830

718 (PRIMER: 5'- ATTTTTGCCGAAGCGCTTGA), Tb927.10.12940 (PRIMER: 5'-

719 CCGTGATTCCCTGTCGACAT), and Tb927.11.15810 (PRIMER: 5'-

720 CACCACCCGATGTACGGTAG). With these primer combinations only transcripts

721 arising from the exogenous ORFeome copy were measured with and without Dox

722 induction (FIG. 3B).

723

724 Next-generation Illumina sequencing of the T. brucei ORFeome and Gain-of-

725 Function libraries

726

Libraries for the pENTR and pDEST (pTrypLib) ORF plasmid libraries were

727 prepared for next generation Illumina sequencing using tagmentation kits from Illumina

728 (Nextera XT kit) according to the manufacturer's instructions. Sequencing was

729 performed either on an Illumina HiSeq 2500 or an Illumina MiSeq. Reads were trimmed

730 for quality using trim galore

731 (http://www.bioinformatics.babraham.ac.uk/projects/trim_galore/) using this command:

732 trim_galore --nextera --stringency 3. Reads were aligned to the Tb927v5.1 genome

733 using bowtie (56) requiring unique alignments: bowtie --best --strata -t -v 2 -a -m 1, or

734 allowing multiple alignments: bowtie --best --strata -t -v 2 -a -m 10. RPKM values were 
735 calculated using SeqMonk from Babraham Bioinformatics

736 (http://www.bioinformatics.babraham.ac.uk/projects/seqmonk).

737 For quantification of introduced ORFs following transfection into the T. brucei to 738 generate the GoF library, genomic DNA was isolated from 100-200 million transfected

739 parasites and fragmented by sonicating water bath (Bioruptor). The NEBNext Ultra II

740 DNA library prep kit was then used to prepare libraries for high throughput sequencing

741 according to the manufacturer's instructions with the following exception: a custom

742 universal primer containing the ATT cloning site was used for 5 ' indexing and PCR

743 enrichment (5'- AATGATACGGCGACCACCGAGATATATAACAAGTTTGTACA

744 AAAAAGCAGGCTATG). A custom sequencing oligo (5'-GGGACAAGTTTGTAC

745 AAAAAAGCAGGCTATG) is loaded on to the Illumina sequencing platform to sequence

746 only GoF library containing reads. Analysis and raw counts were calculated using

747 SeqMonk from Babraham Bioinformatics

748 (http://www.bioinformatics.babraham.ac.uk/projects/seqmonk). Sequencing reads were

749 trimmed for quality and aligned to the genome using the same parameters described

750 above. Raw counts were calculated for reads aligning exclusively to the first $100 \mathrm{bp}$ of

751 the ORF using SeqMonk from Babraham Bioinformatics. DESeq2 was used to calculate

752 the normalized read counts between sequencing libraries (38).

753

\section{Melarsoprol GoF genetic screening}

755 For melarsoprol selective screening, GoF library cells were seeded for each condition at

$7561 \times 10^{5}$ cell $/ \mathrm{ml}$, induced with doxycycline $(1 \mu \mathrm{g} / \mathrm{mL}) 24$ hours (when appropriate), and

757 grown in HMl-9 medium containing Dox (when appropriate) plus melarsoprol at $17 \mathrm{nM}$ 
758 or 35 nM melarsoprol (BoC sciences, CAS 494-79-1). Melarsoprol stocks were diluted in 759 DMSO.

\section{Bioinformatic analysis platforms for ORF identification and hit calling pipeline}

In order to call which genes were overrepresented in the melarsoprol selected

libraries, we exclusively counted reads that fell within the first $100 \mathrm{bp}$ of the gene. DeSeq

(38) was then used to identify genes that were 'differentially expressed' in the

765

melarsoprol selected libraries with a multiple testing corrected $p$ value of less than 0.05 .

766

Using normalized read counts, we then calculated the fold change between each pair of

melarsoprol selected and unselected replicates (3 total) for every statistically significant

768

gene called by DESeq, provided that gene had a normalized read count of 5 or greater

769

in the minimally propagated samples. If all 3 replicates showed a $>4$ fold change for a

particular gene, that gene was considered significantly overrepresented. Because we

771 had two sets of replicates for unselected libraries and two sets of replicates for

772 melarsoprol selected libraries, we ran the pipeline described above for all 4

773 comparisons of selected and unselected sets of replicates. Only those genes that were

774 called as hits in all 4 comparisons were reported in the final hit list.

775

776

\section{EC50 determination by alamarblue}

777 For EC50 determination, induced and uninduced cells were plated across a melarsoprol

778 dilution series and assessed viability after 72 hours using Alamarblue (ThermoFisher)

779 as previously described (57). All experiments were performed in biological triplicate.

\section{ACKNOWLEDGEMENTS}


The authors would like to extend our sincere thanks to the individuals and consortiums whose support made this work possible. Dr. Marilyn Parsons (now of Seattle Children's Hospital) provided prepublication access to the TREU927 ribosomal profiling data that provided the gene starts and stops for all ORFeome targeted ORFs.

Drs. Christine Clayton and Esteban Erben (Heidelberg University) provided their essential insights into methods for successful ORFeome gateway cloning. Data provided directly from the tryptag.org consortium was critical in the categorization of hits arising from melarsoprol GoF screening. Similarly, tritrypdb.org was an essential resource throughout all stages of the work described herein. We thank Eugenia SilvaHerzog for her contribution to the generation of 'mini-library' overexpression data. Finally, we would like to thank Dr. F. Nina Papavasiliou, whose support and generosity have been invaluable.

\section{REFERENCES}

795

1. Barrett MP, Burchmore RJS, Stich A, Lazzari JO, Frasch AC, Cazzulo JJ, Krishna

2. Fairlamb AH, Gow NAR, Matthews KR, Waters AP. Drug resistance in eukaryotic microorganisms. Nature Microbiology. 2016 Jun 24;1(7):555-15. PMCID: PMC5215055

3. El-Sayed NM, Myler PJ, Blandin G, Berriman M, Crabtree J, Aggarwal G, Caler E, Renauld H, Worthey EA, Hertz-Fowler C, Ghedin E, Peacock C, Bartholomeu DC, Haas BJ, Tran A-N, Wortman JR, Alsmark UCM, Angiuoli S, Anupama A, Badger J, Bringaud F, Cadag E, Carlton JM, Cerqueira GC, Creasy T, Delcher AL, Djikeng A, Embley TM, Hauser C, Ivens AC, Kummerfeld SK, Pereira-Leal JB, Nilsson D, Peterson J, Salzberg SL, Shallom J, Silva JC, Sundaram J, Westenberger S, White O, Melville SE, Donelson JE, Andersson B, Stuart KD, Hall N. Comparative genomics of trypanosomatid parasitic protozoa. Science (New York, N.Y.). American Association for the Advancement of Science; 2005 Jul 15;309(5733):404-9. 
811

812

813

814

815

816

817

818

819

820

821

822

823

824

825

826

827

828

829

830

831

832

833

834

835

836

837

838

839

840

841

842

843

844

845

846

847

4. Akiyoshi B, Gull K. Evolutionary cell biology of chromosome segregation: insights from trypanosomes. Open Biol. 2013 May;3(5):130023. PMCID: PMC3866873

5. Matthews KR. 25 years of African trypanosome research: From description to molecular dissection and new drug discovery. Molecular \& Biochemical Parasitology. 2015 Mar;200(1-2):30-40. PMCID: PMC4509711

6. Mani J, Meisinger C, Schneider A. Peeping at TOMs-Diverse Entry Gates to Mitochondria Provide Insights into the Evolution of Eukaryotes. Mol. Biol. Evol. 2016 Feb;33(2):337-51.

7. Jackson AP, Otto TD, Aslett M, Armstrong SD, Bringaud F, Schlacht A, Hartley C, Sanders M, Wastling JM, Dacks JB, Acosta-Serrano A, Field MC, Ginger ML, Berriman M. Kinetoplastid Phylogenomics Reveals the Evolutionary Innovations Associated with the Origins of Parasitism. Curr. Biol. 2016 Jan 25;26(2):161-72. PMCID: PMC4728078

8. Mayor S, Menon AK, Cross GA. Glycolipid precursors for the membrane anchor of Trypanosoma brucei variant surface glycoproteins. II. Lipid structures of phosphatidylinositol-specific phospholipase $\mathrm{C}$ sensitive and resistant glycolipids. J. Biol. Chem. 1990 Apr 15;265(11):6174-81.

9. Tschudi C, Ullu E. Trypanosomatid protozoa provide paradigms of eukaryotic biology. Infect Agents Dis. 1994 Aug;3(4):181-6.

10. Simpson L, Thiemann OH, Savill NJ, Alfonzo JD, Maslov DA. Evolution of RNA editing in trypanosome mitochondria. Proc Natl Acad Sci USA. National Academy of Sciences; 2000 Jun 20;97(13):6986-93. PMCID: PMC34374

11. Alsford S, KELLY JM, Baker N, Horn D. Genetic dissection of drug resistance in trypanosomes. Parasitology. George Washington Libraries; 2013 Apr 3;140(12):1478-91.

12. Glover L, Alsford S, Baker N, Turner DJ, Sanchez-Flores A, Hutchinson S, HertzFowler C, Berriman M, Horn D. Genome-scale RNAi screens for high-throughput phenotyping in bloodstream-form African trypanosomes. Nat Protoc. 2015 Jan;10(1):106-33.

13. Currier RB, Cooper A, Burrell-Saward H, Macleod A, Alsford S. Decoding the network of Trypanosoma brucei proteins that determines sensitivity to apolipoprotein-L1. Raper J, editor. PLoS Pathog. 2018 Jan 18;14(1):e100685526.

14. Alsford S, Turner DJ, Obado SO, Sanchez-Flores A, Glover L, Berriman M, HertzFowler C, Horn D. High-throughput phenotyping using parallel sequencing of RNA interference targets in the African trypanosome. Genome Research. Cold Spring Harbor Lab; 2011 Jun 1;21(6):915-24. PMCID: PMC3106324 
15. Normark S, Edlund T, Grundström T, Bergström S, Wolf-Watz H. Escherichia coli K-12 mutants hyperproducing chromosomal beta-lactamase by gene repetitions. J. Bacteriol. American Society for Microbiology (ASM); 1977 Dec;132(3):912-22. PMCID: PMC235595

16. Rine J, Hansen W, Hardeman E, Davis RW. Targeted selection of recombinant clones through gene dosage effects. Proc Natl Acad Sci USA. National Academy of Sciences; 1983 Nov;80(22):6750-4. PMCID: PMC390063

17. Sandegren L, Andersson DI. Bacterial gene amplification: implications for the evolution of antibiotic resistance. Nat. Rev. Microbiol. Nature Publishing Group; 2009 Aug;7(8):578-88.

18. Prelich G. Gene overexpression: uses, mechanisms, and interpretation. Genetics. Genetics; 2012 Mar;190(3):841-54. PMCID: PMC3296252

19. Clayton CE. Gene expression in Kinetoplastids. Curr. Opin. Microbiol. 2016 Aug;32:46-51.

20. Begolo D, Erben E, Clayton C. Drug target identification using a trypanosome overexpression library. Antimicrob. Agents Chemother. American Society for Microbiology Journals; 2014 Oct;58(10):6260-4. PMCID: PMC4187942

21. Wall RJ, Rico E, Lukac I, Zuccotto F, Elg S, Gilbert IH, Freund Y, Alley MRK, Field MC, Wyllie S, Horn D. Clinical and veterinary trypanocidal benzoxaboroles target CPSF3. Proc. Natl. Acad. Sci. U.S.A. National Academy of Sciences; 2018 Sep 18;115(38):9616-21. PMCID: PMC6156652

22. Rual J-F, Hill DE, Vidal M. ORFeome projects: gateway between genomics and omics. Curr Opin Chem Biol. 2004 Feb;8(1):20-5.

23. Li Q-R, Carvunis A-R, Yu H, Han J-DJ, Zhong Q, Simonis N, Tam S, Hao T, Klitgord NJ, Dupuy D, Mou D, Wapinski I, Regev A, Hill DE, Cusick ME, Vidal M. Revisiting the Saccharomyces cerevisiae predicted ORFeome. Genome Research. Cold Spring Harbor Lab; 2008 Aug;18(8):1294-303. PMCID: PMC2493439

24. Gallegos ME, Balakrishnan S, Chandramouli P, Arora S, Azameera A, Babushekar A, Bargoma E, Bokhari A, Chava SK, Das P, Desai M, Decena D, Saramma SDD, Dey B, Doss A-L, Gor N, Gudiputi L, Guo C, Hande S, Jensen M, Jones S, Jones N, Jorgens D, Karamchedu P, Kamrani K, Kolora LD, Kristensen L, Kwan K, Lau H, Maharaj P, Mander N, Mangipudi K, Menakuru H, Mody V, Mohanty S, Mukkamala S, Mundra SA, Nagaraju S, Narayanaswamy R, NdunguCase C, Noorbakhsh M, Patel J, Patel P, Pendem SV, Ponakala A, Rath M, Robles MC, Rokkam D, Roth C, Sasidharan P, et al. The C. elegans rab family: identification, classification and toolkit construction. D'Adamo P, editor. PLoS ONE. 2012;7(11):e49387. PMCID: PMC3504004 
25. Mehla J, Caufield JH, Uetz P. Mapping Protein-Protein Interactions Using Yeast Two-Hybrid Assays. Cold Spring Harb Protoc. 2015 May 1;2015(5):pdb.prot086157-12.

26. Bischof J, Duffraisse M, Furger E, Ajuria L, Giraud G, Vanderperre S, Paul R, Björklund M, Ahr D, Ahmed AW, Spinelli L, Brun C, Basler K, Merabet S. Generation of a versatile BiFC ORFeome library for analyzing protein-protein interactions in live Drosophila. Elife. eLife Sciences Publications Limited; 2018 Sep 24;7:1251. PMCID: PMC6177257

27. King J, Foster J, Davison JM, Rawls JF, Breton G. Zebrafish Transcription Factor ORFeome for Gene Discovery and Regulatory Network Elucidation. Zebrafish. Mary Ann Liebert, Inc. 140 Huguenot Street, 3rd Floor New Rochelle, NY 10801 USA; 2018 Apr;15(2):202-5. PMCID: PMC6112160

28. Trindade S, Rijo-Ferreira F, Carvalho T, Pinto-Neves D, Guegan F, Aresta-Branco F, Bento F, Young SA, Pinto A, Van Den Abbeele J, Ribeiro RM, Dias S, Smith TK, Figueiredo LM. Trypanosoma brucei Parasites Occupy and Functionally Adapt to the Adipose Tissue in Mice. Cell Host Microbe. Elsevier Inc; 2016 Jun 8;19(6):837-48.

29. Deeks ED. Fexinidazole: First Global Approval. Drugs. 2019 Feb;79(2):215-20.

30. Fairlamb AH, Horn D. Melarsoprol Resistance in African Trypanosomiasis. Elsevier Ltd; 2018 May 10;:1-12.

31. Krauth-Siegel RL, Comini MA. Redox control in trypanosomatids, parasitic protozoa with trypanothione-based thiol metabolism. Biochimica et Biophysica Acta (BBA) - General Subjects. 2008 Nov;1780(11):1236-48.

32. Parsons M, Ramasamy G, Vasconcelos EJR, Jensen BC, Myler PJ. Advancing Trypanosoma brucei genome annotation through ribosome profiling and spliced leader mapping. Molecular \& Biochemical Parasitology. 2015 Aug;202(2):1-10. PMCID: PMC4644489

33. Shahi SK, Krauth-Siegel RL, Clayton CE. Overexpression of the putative thiol conjugate transporter TbMRPA causes melarsoprol resistance in Trypanosoma brucei. Mol. Microbiol. John Wiley \& Sons, Ltd (10.1111); 2002 Mar;43(5):1129_ 38.

34. Graf FE, Ludin P, Wenzler T, Kaiser M, Brun R, Pyana PP, Büscher P, de Koning HP, Horn D, Mäser P. Aquaporin 2 Mutations in Trypanosoma brucei gambiense Field Isolates Correlate with Decreased Susceptibility to Pentamidine and Melarsoprol. Matovu E, editor. PLoS Negl Trop Dis. 2013 Oct 10;7(10):e2475-7. PMCID: PMC3794916 
35. Baker N, de Koning HP, Mäser P, Horn D. Drug resistance in Africantrypanosomiasis: the melarsoprol andpentamidine story. Trends Parasitol. Elsevier Ltd; 2013 Mar 1;29(3):110-8.

36. Munday JC, Eze AA, Baker N, Glover L, Clucas C, Aguinaga Andrés D, Natto MJ, Teka IA, McDonald J, Lee RS, Graf FE, Ludin P, Burchmore RJS, Turner CMR, Tait A, Macleod A, Mäser P, Barrett MP, Horn D, de Koning HP. Trypanosoma brucei aquaglyceroporin 2 is a high-affinity transporter for pentamidine and melaminophenyl arsenic drugs and the main genetic determinant of resistance to these drugs. J. Antimicrob. Chemother. 2014 Mar;69(3):651-63. PMCID: PMC3922157

37. Graf FE, Ludin P, Arquint C, Schmidt RS, Schaub N, Renggli CK, Munday JC, Krezdorn J, Baker N, Horn D, Balmer O, Caccone A, Koning HP, Mäser P. Comparative genomics of drug resistance in Trypanosoma brucei rhodesiense. Cellular and Molecular Life Sciences. Springer International Publishing; 2016 Mar 13;73(17):3387-400. PMCID: PMC4967103

38. Love MI, Huber W, Anders S. Moderated estimation of fold change and dispersion for RNA-seq data with DESeq2. Genome Biol. BioMed Central; 2014;15(12):550-21. PMCID: PMC4302049

39. Lueder DV, Phillips MA. Characterization of Trypanosoma brucei gammaglutamylcysteine synthetase, an essential enzyme in the biosynthesis of trypanothione (diglutathionylspermidine). J. Biol. Chem. American Society for Biochemistry and Molecular Biology; 1996 Jul 19;271(29):17485-90.

40. Huynh TT, Huynh VT, Harmon MA, Phillips MA. Gene Knockdown of YGlutamylcysteine Synthetase by RNA in the Parasitic Protozoa Trypanosoma bruceiDemonstrates That It Is an Essential Enzyme. J. Biol. Chem. 2003 Oct 3;278(41):39794-800.

41. Singh A, Minia I, Droll D, Fadda A, Clayton C, Erben E. Trypanosome MKT1 and the RNA-binding protein ZC3H11: interactions and potential roles in posttranscriptional regulatory networks. Nucleic Acids Res. 2014 Jan 25;42(7):465268.

42. Alsford S, Turner DJ, Obado SO, Sanchez-Flores A, Glover L, Berriman M, HertzFowler C, Horn D. High-throughput phenotyping using parallel sequencing of RNA interference targets in the African trypanosome. Genome Research. 2011 Jun 1;21(6):915-24. PMCID: PMC3106324

43. Motyka SA, Drew ME, Yildirir G, Englund PT. Overexpression of a cytochrome b5 reductase-like protein causes kinetoplast DNA loss in Trypanosoma brucei. J. Biol. Chem. American Society for Biochemistry and Molecular Biology; $2006 \mathrm{Jul}$ 7;281(27):18499-506. 
960

44. Thomas JA, Baker N, Hutchinson S, Dominicus C, Trenaman A, Glover L, Alsford $S$, Horn D. Insights into antitrypanosomal drug mode-of-action from cytologybased profiling. Clos J, editor. PLoS Negl Trop Dis. Public Library of Science; 2018 Nov 26;12(11):e0006980-19.

45. Alsford S, Eckert S, Baker N, Glover L, Sanchez-Flores A, Leung KF, Turner DJ, Field MC, Berriman M, Horn D. High-throughput decoding of antitrypanosomal drug efficacy and resistance. Nature. Nature Publishing Group; 2019 Apr 24;482(7384):232-6.

46. Baker N, Glover L, Munday JC, Aguinaga Andrés D, Barrett MP, de Koning HP, Horn D. Aquaglyceroporin 2 controls susceptibility to melarsoprol and pentamidine in African trypanosomes. Proc. Natl. Acad. Sci. U.S.A. National Academy of Sciences; 2012 Jul 3;109(27):10996-1001. PMCID: PMC3390834

47. Fidalgo LM, Gille L. Mitochondria and Trypanosomatids: Targets and Drugs. Pharm Res. 2011 Sep 21;28(11):2758-70.

48. Stephens JL, Lee SH, Paul KS, Englund PT. Mitochondrial fatty acid synthesis in Trypanosoma brucei. J. Biol. Chem. 2007 Feb 16;282(7):4427-36.

49. Grant IM, Balcha D, Hao T, Shen Y, Trivedi P, Patrushev I, Fortriede JD, Karpinka JB, Liu L, Zorn AM, Stukenberg PT, Hill DE, Gilchrist MJ. The Xenopus ORFeome: A resource that enables functional genomics. Dev. Biol. 2015 Dec 15;408(2):345-57. PMCID: PMC4684507

50. Mehla J, Caufield JH, Uetz P. Mapping Protein-Protein Interactions Using Yeast Two-Hybrid Assays. Cold Spring Harb Protoc. 2015 May 1;2015(5):pdb.prot086157-12.

51. Alsford S, Eckert S, Baker N, Glover L, Sanchez-Flores A, Leung KF, Turner DJ, Field MC, Berriman M, Horn D. High-throughput decoding of antitrypanosomal drug efficacy and resistance. Nature. Nature Publishing Group; 2012 Jan 25;482(7384):232-6. PMCID: PMC3303116

52. Siegel TN, Tan KSW, Cross GAM. Systematic study of sequence motifs for RNA trans splicing in Trypanosoma brucei. Mol. Cell. Biol. 2005 Nov;25(21):9586-94. PMCID: PMC1265811

53. Wirtz E, Leal S, Ochatt C, Cross GA. A tightly regulated inducible expression system for conditional gene knock-outs and dominant-negative genetics in Trypanosoma brucei. Molecular \& Biochemical Parasitology. 1999 Mar 15;99(1):89-101.

54. Hirumi H, Hirumi K. Axenic culture of African trypanosome bloodstream forms. Parasitol. Today (Regul. Ed.). 1994 Feb;10(2):80-4. 
996

1006

1007

1008

1009

1010

1011

1012

1013

1014

1015

1016

1017

1018

1019

1020

1021
55. Burkard G, Fragoso CM, Roditi I. Highly efficient stable transformation of bloodstream forms of Trypanosoma brucei. Molecular \& Biochemical Parasitology. 2007 Jun;153(2):220-3.

56. Langmead B, Trapnell C, Pop M, Salzberg SL. Ultrafast and memory-efficient alignment of short DNA sequences to the human genome. Genome Biol. BioMed Central; 2009;10(3):R25-10. PMCID: PMC2690996

57. Baker N, Alsford S, Horn D. Genome-wide RNAi screens in African trypanosomes identify the nifurtimox activator NTR and the eflornithine transporter AAT6.

Molecular \& Biochemical Parasitology. Elsevier B.V; 2011 Mar 1;176(1):55-7.

PMCID: PMC3032052 


\section{FIGURES AND FIGURE LEGENDS}

1024

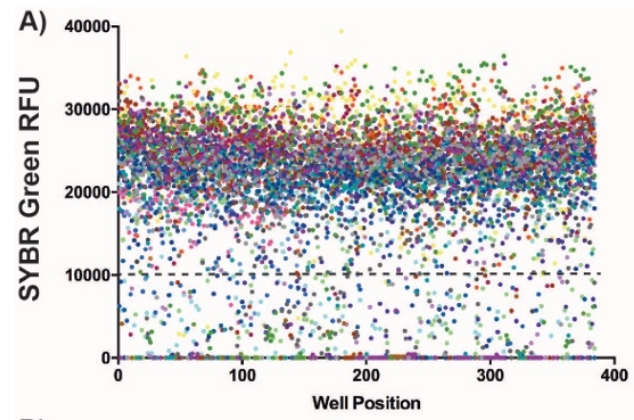

D) ORF Identifcation
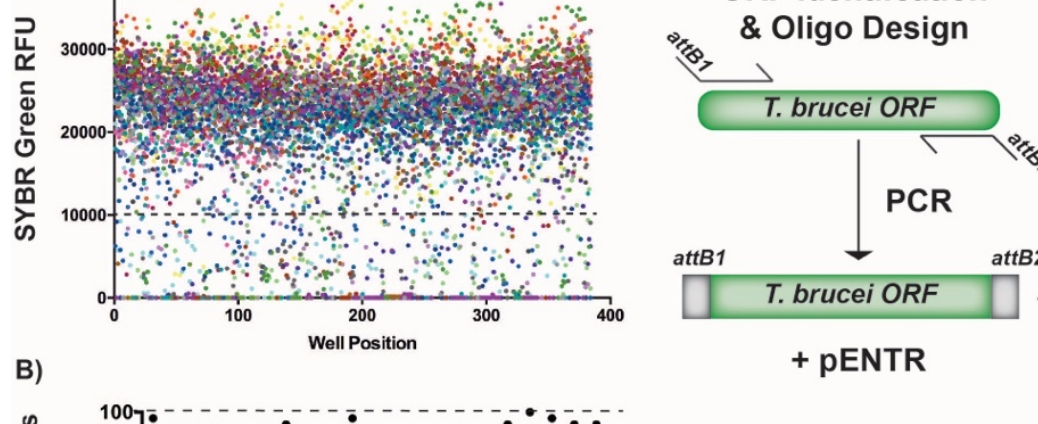

+ pENTR

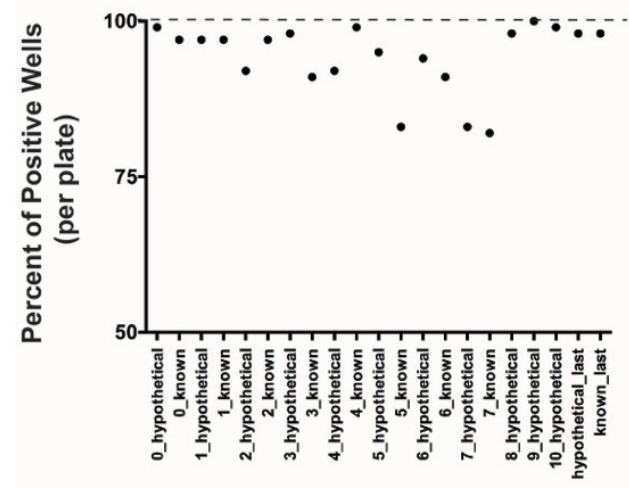

C)
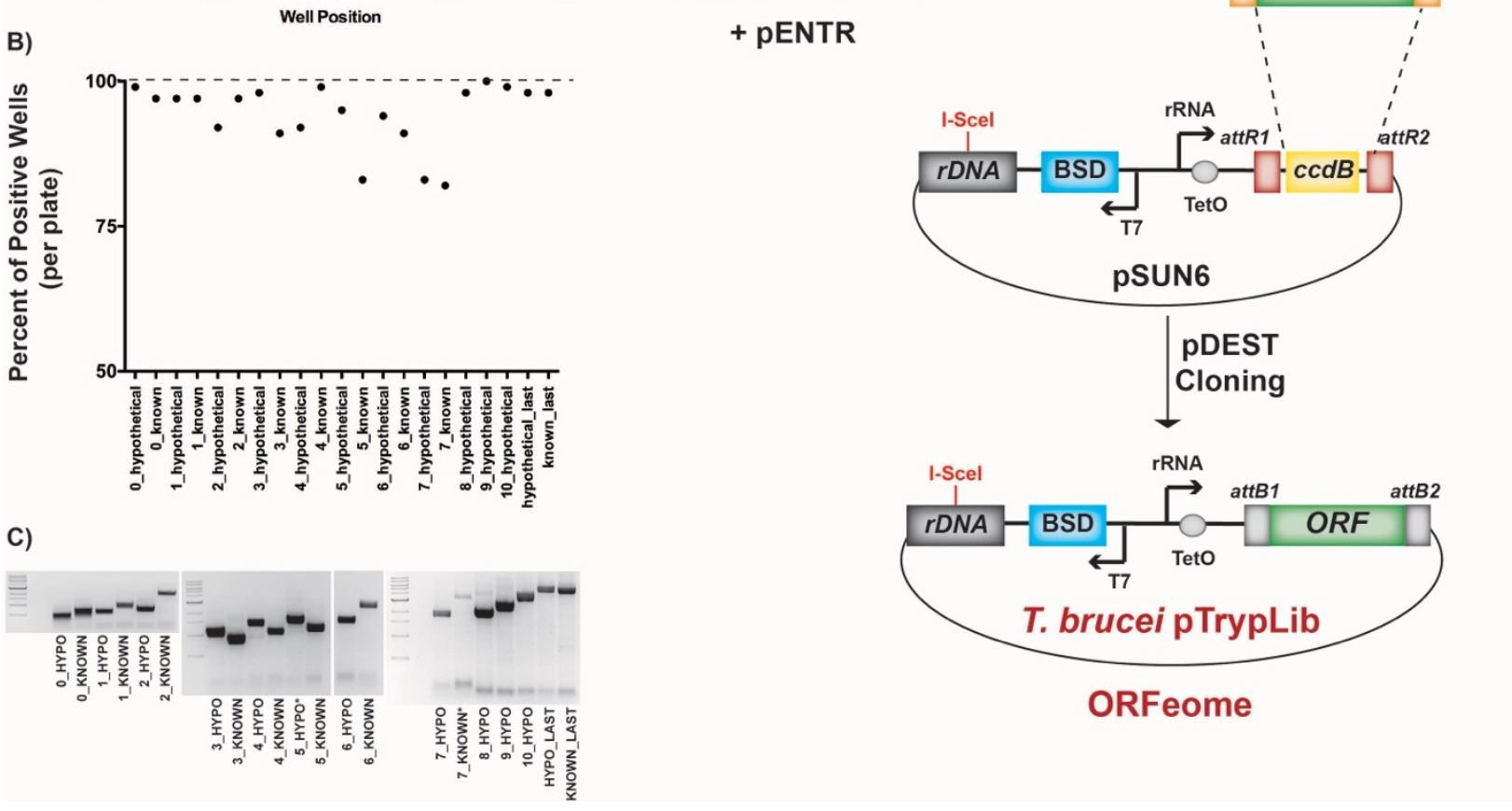

1025

Figure 1. Generating a T. brucei ORFeome. A) Assessment of PCR amplification by

1027 SYBR Green Relative Fluorescence Units (RFU). Each dot represents an individual

1028 PCR reaction, each color represents one of the 21, 384-well plates, graph is of SYBR

1029 Relative Fluorescence Units (RFU) vs. 384-well plate position. B) Percent of PCR

1030 positive wells (SYBR assessment) for each of the 21, 384-well plates, from the first time

1031 amplified. C) Agarose gel bands from each of the 21, 384-well PCR plates pooled prior

1032 to gel extraction and cloning, from the first time amplified, compared to $10 \mathrm{~Kb}$ ladder

1033 DNA ladder. D) ORFeome cloning strategy: attB1 site addition to T. brucei ORFs during 
1034 PCR amplification, Gateway cloning into pENTR to generate the pENTR ORFeome, 1035 and Gateway cloning into T. brucei specific pDEST type vector (pSUN6, shown in detail 1036 in SUP. 3) to generate the complete pTrypLib ORFeome.

A)

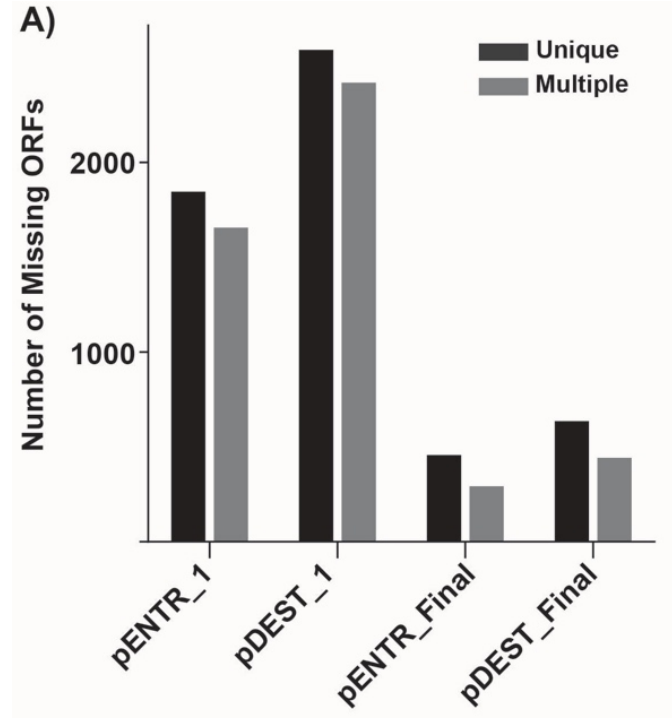

B)

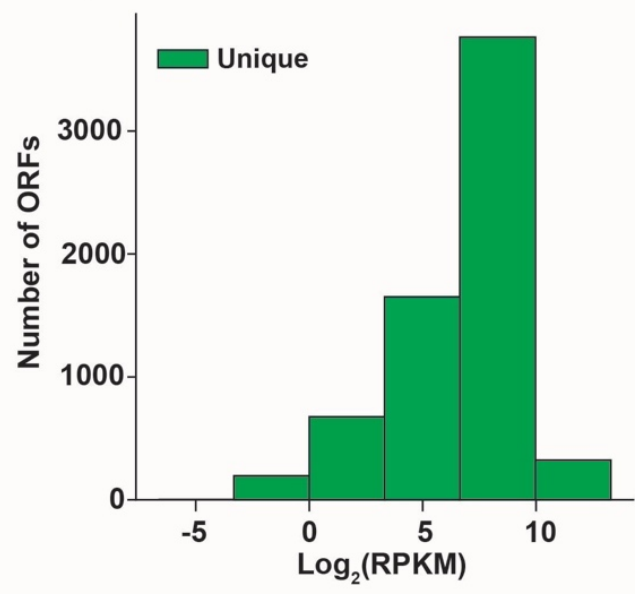

C)

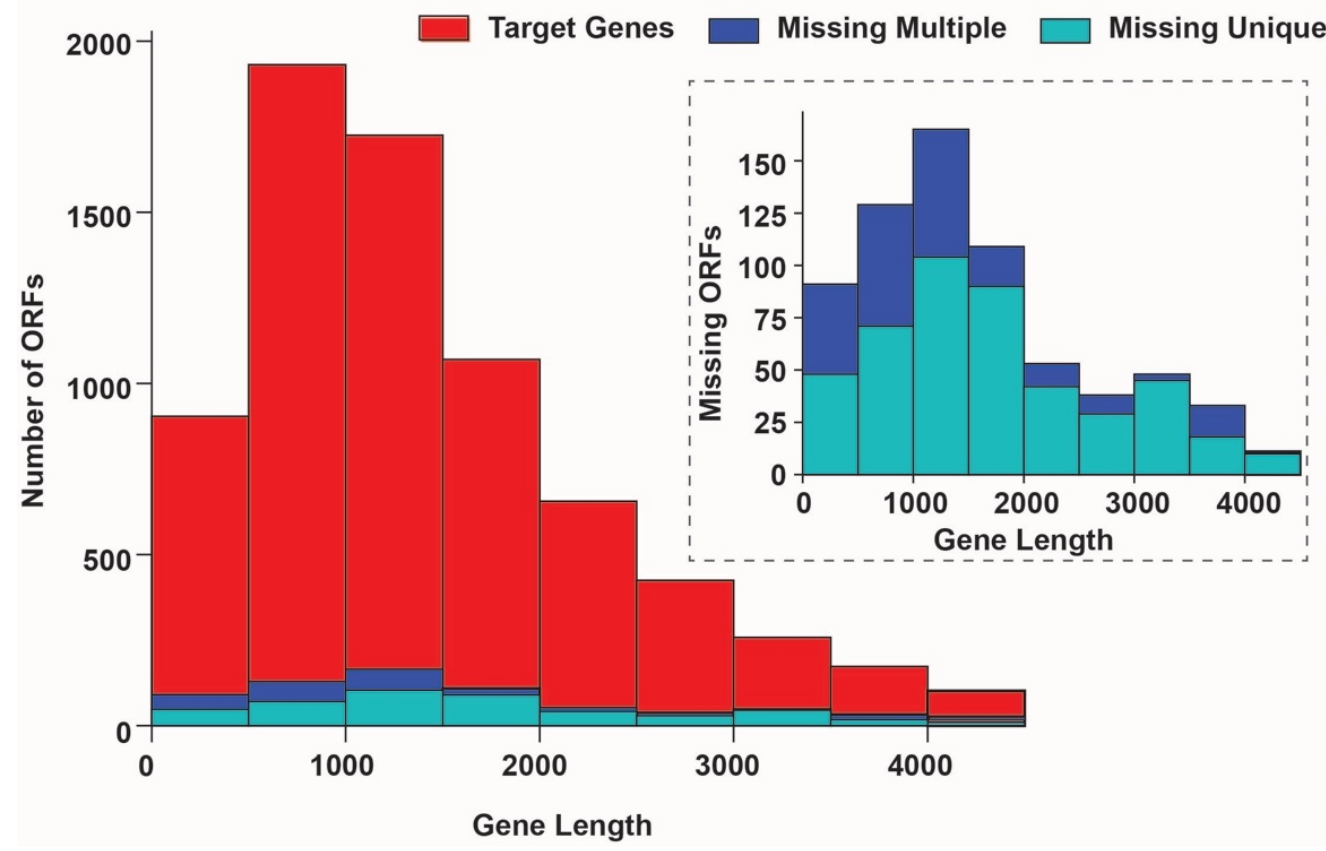

Figure 2. Assessment of pENTR and pDEST (pTrypLib) plasmid libraries. A) Bar

1040 graph showing the number of targeted ORFs with zero detectable aligned reads from 
1041 the first round of cloning (pENTR_1 and pDEST_1) and after both rounds of cloning 1042 (pENTR_Final and pDEST_Final) using analysis generated from both uniquely and 1043 multiply aligned reads. B) Histogram showing the distribution of normalized read counts 1044 for each ORF in the pooled pDEST (pTrypLib) plasmid libraries (Uniquely aligned reads 1045 shown, SUP. 6 both uniquely and multiply aligned reads). C) Histograms showing the 1046 distribution of ORF lengths for the target gene list (red) and the set of ORFs with zero 1047 detectable aligned reads after both rounds of cloning (labeled as missing). Analyses

1048 from unique (dark blue) and multiply (light blue) aligned reads are shown. Inset graph, 1049 target ORF lengths have been left out to better visualize the lengths of the missing 1050 ORFs.

1051

1052 
A)
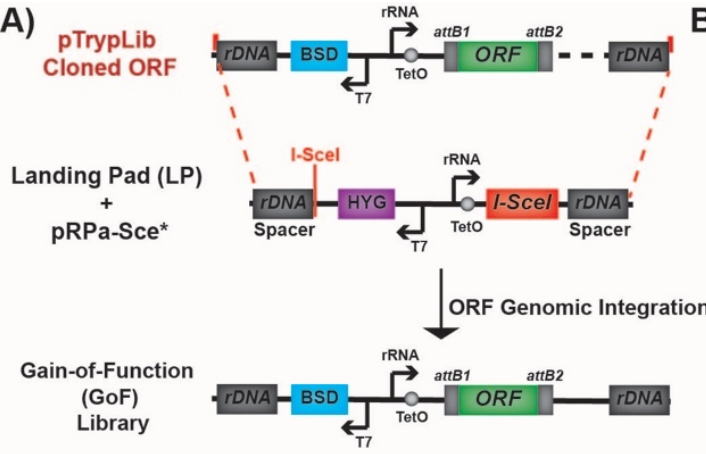

C)

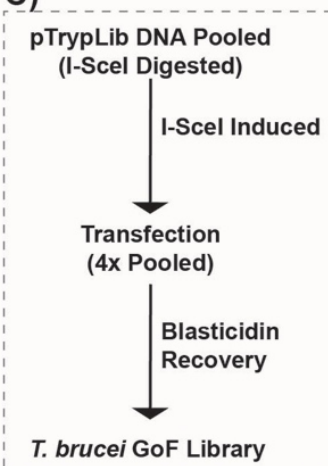

1054

1055

1056

1057

1058

1059

1060

1061

1062

1063

1064

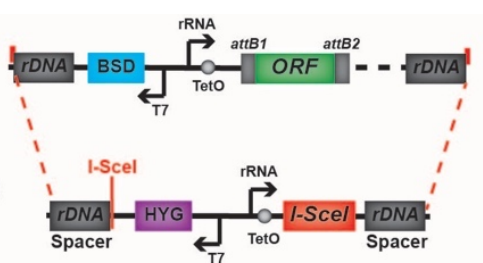

B) 6 Colonies Picked

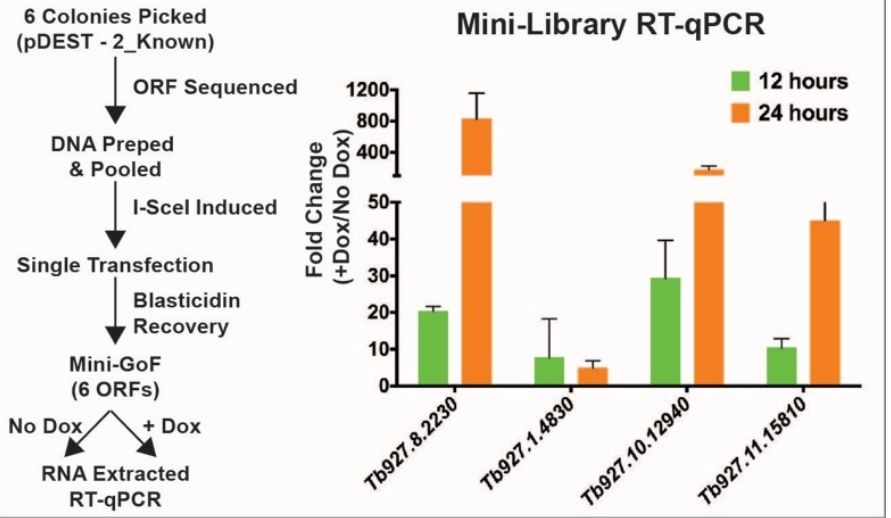

RT-qPCR

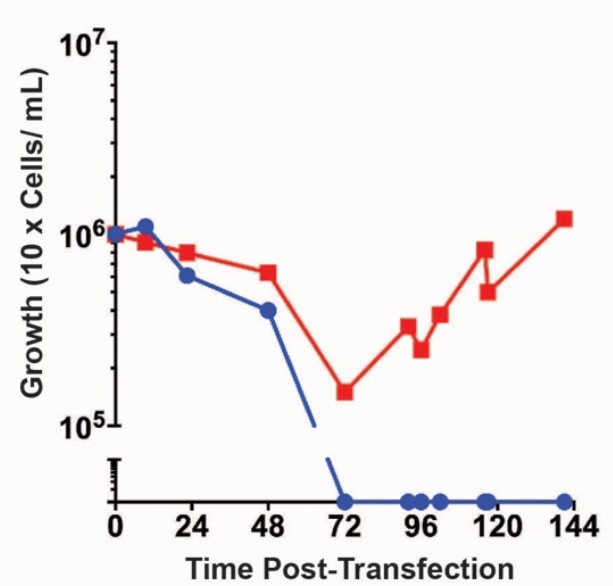

(Hours)
D)

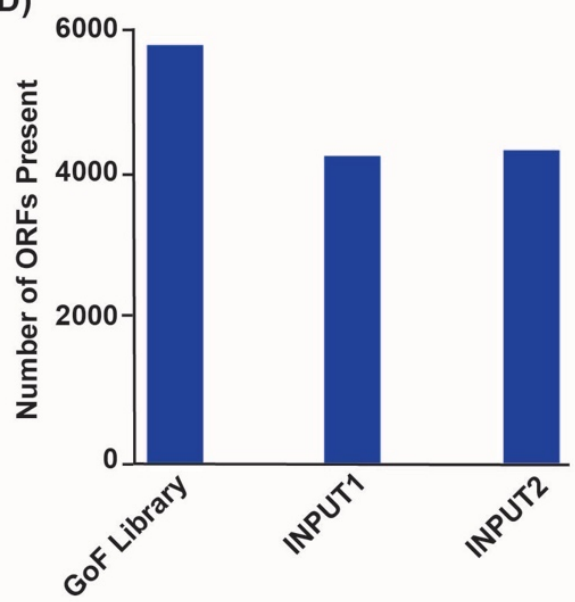

Figure 3. Generation and validation of the T. brucei GoF library. A) Transfection of pTrypLib ORFeome into parental Landing Pad (LP) cell line harboring pRPa-Sce* plasmid for I-Scel induced enzymatic cleavage of a single rDNA spacer site (12). B) Generation of a Mini-library GoF library, 6 ORFs. Procedure shown in flow diagram on the left. Transcript level of four GoF library encoded ORFs (exogenous) measured by RT-qPCR following 12 and 24 hours of doxycycline induction compared to uninduced cells (No Dox). C) Generation of the pTrypLib ORFeome based GoF library. Procedure shown in flow diagram on the left. Graph indicates the recovery of GoF library harboring cells (red line) compared to mock transfection (blue line) in blasticidin. D) Assessment of the number of ORFeome genes present in the GoF library following initial transfection 
1065 (GoF library), in INPUT1 (following freeze thaw and minimal propagation), and INPUT2, 1066 which used an alternative NGS protocol.

1067
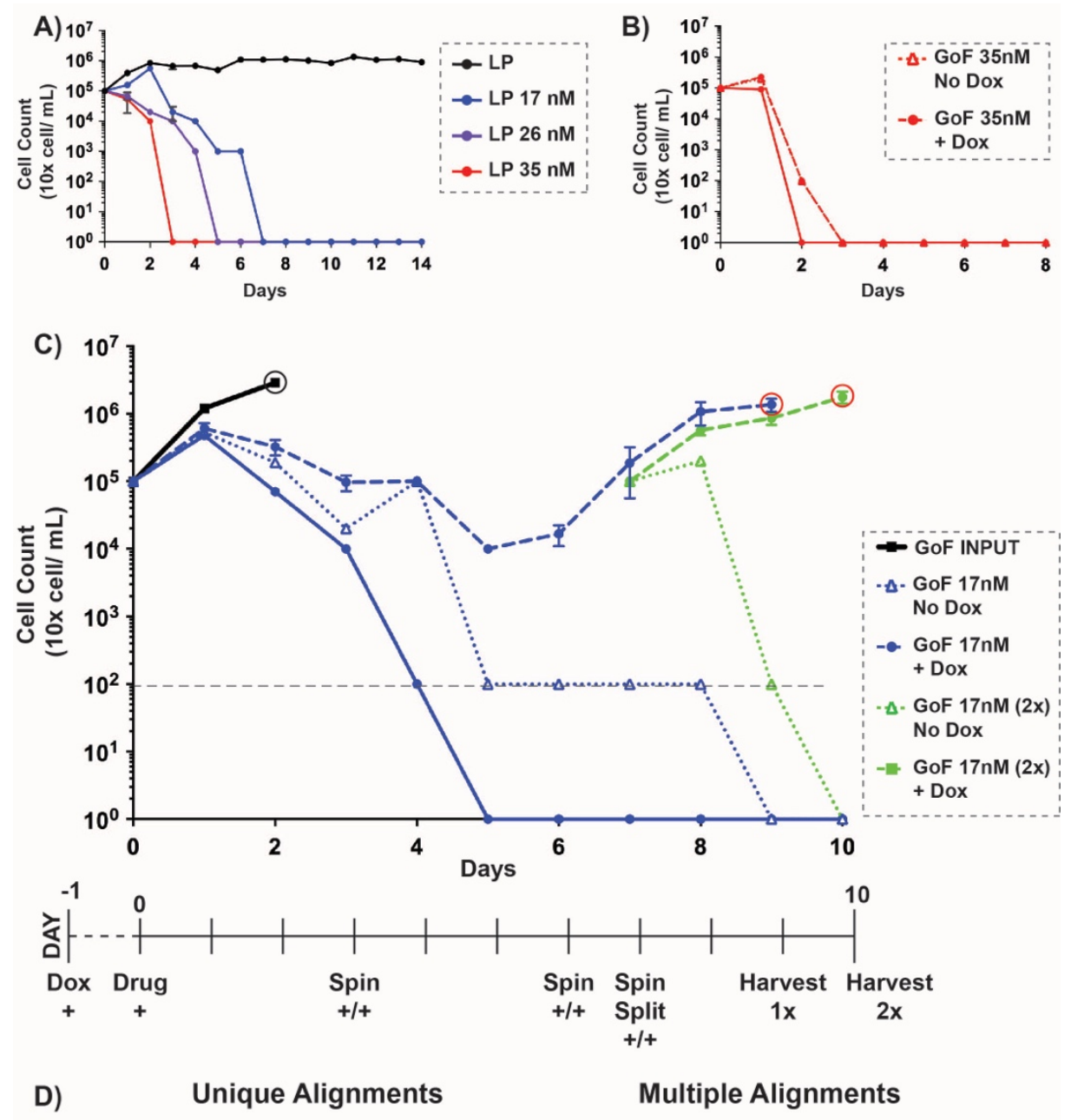

Multiple Alignments
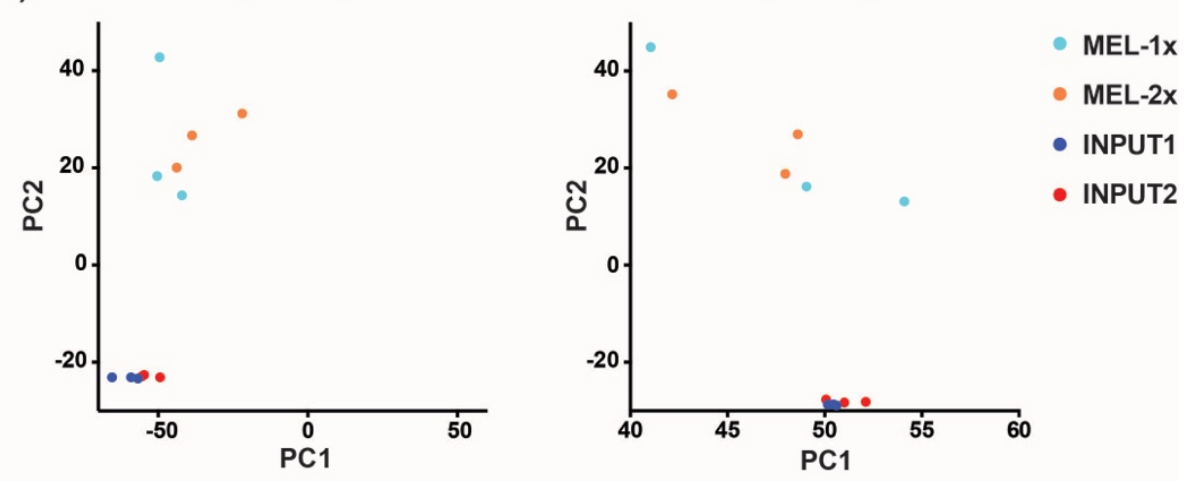

1070 Growth of Landing Pad (LP) parental cell line in 17 nM (blue line), 26 nM (purple line), 
$107135 \mathrm{nM}$ (red line), or no (black line) melarsoprol treatment over 14 days. B) GoF library

1072 screen in 35 nM melarsoprol treatment: LP cell line (solid red line), uninduced GoF

1073 library (red dotted line), induced GoF library (red dashed line); dotted and dashed lines

1074 overlap. C) GoF genetic screen in $17 \mathrm{nM}$ melarsoprol is shown as a time course at the

1075 bottom and graph of cell growth: INPUT, untreated GoF library harboring cells grown for

10763 days (black line), LP parental cell line (solid blue line), uninduced GoF library (blue

1077 triangles on dotted line), induced GoF library (blue circles on dashed line), harvested on

1078 Day 9 (red circle on blue line) to produce MEL-1x. On day 7, biological triplicates from

1079 induced GoF library (blue circles on dashed line) were split into two sets of triplicate

1080 samples, both with $17 \mathrm{nM}$ melarsoprol, one of which was not further induced (green

1081 triangles on dotted line). The other continued to be induced (green squares on dashed

1082 green line) and was harvested on Day 10 to produce MEL-2x (red circle indicates

1083 harvest). D) Principle Component Analysis (PCA) comparing INPUT libraries (1 and 2)

1084 with libraries arising following 2 weeks of continuous melarsoprol selection (MEL-1x and

1085 MEL-2x). 
A)

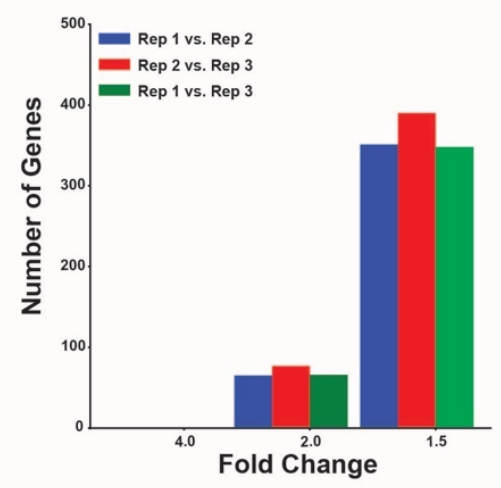

C)

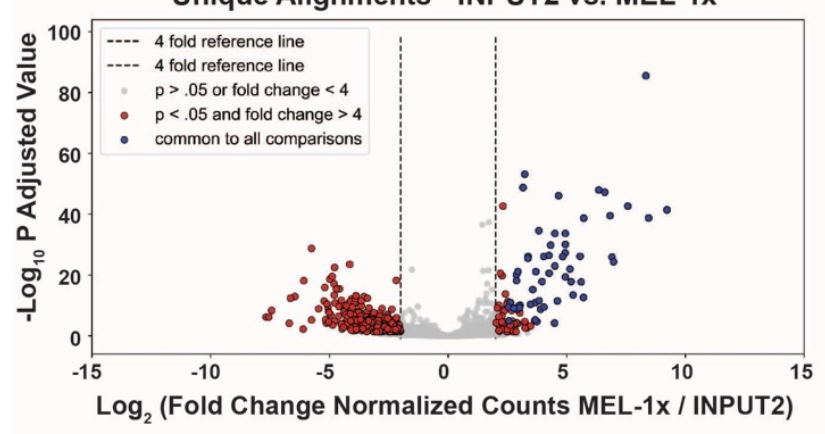

B)

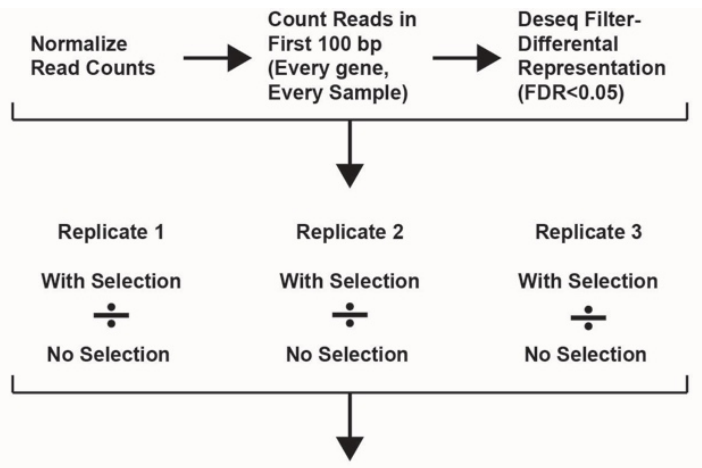

D) Genes Common to Four Comparisions (Unique alignments, Greater than 4-Fold)

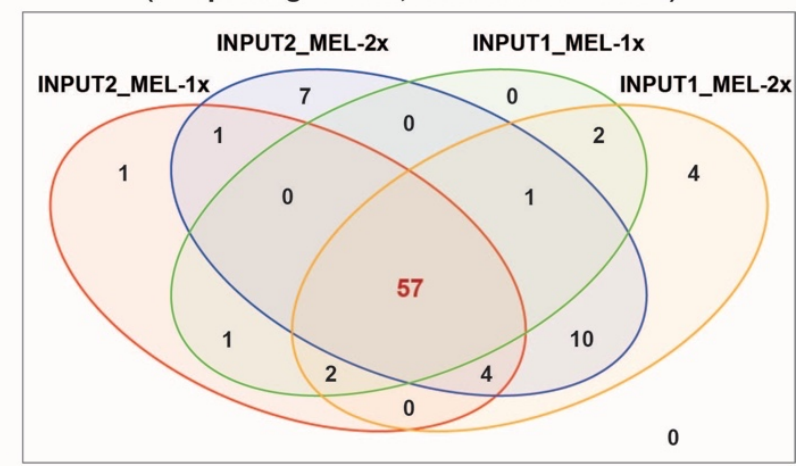

Figure 5. Identification of significantly overrepresented ORFs in melarsoprol GoF survivor populations. A) The number of genes with a fold change $>1.5,2$, or 4 -fold is given for all three replicates of INPUT2: Replicate 1 vs. Replicate 2, Replicate 2 vs. Replicate 3, and Replicate 1 vs. Replicate 3. B) Hit calling pipeline to identify genes overrepresented in melarsoprol survivor populations. Following read count normalization and alignment to the first $100 \mathrm{bp}$ of each gene in every sample, DESeq was used to filter for differential representation using a $\mathrm{P}$ adjusted cutoff of $<0.05$. Each melarsoprol treated replicate was compared with each untreated INPUT replicate and those with 4-fold or greater change of melarsoprol selected over unselected in all three

1097 comparisons were called as preliminary hits. Preliminary hits were determined for INPUT1 vs. MEL-1x, INPUT2 vs. MEL-1x, INPUT1 vs. MEL-2x, and INPUT2 vs. MELplot showing the $-\log _{10}\left(P\right.$ adjusted value) vs. $\log _{2}$ (Fold Change in normalized counts 
1101 for the comparison of melarsoprol selected MEL-1x/INPUT2) for each ORF in the

1102 targeted library. Red dots represent fold changes $>4$ with a P adjusted significance of

$1103<0.05$ and blue dots represent overrepresented ORFs common to all four comparisons

1104 described in B. D) Venn Diagram illustrates the significantly overrepresented genes

1105 common to each comparison and shared among all comparisons, resulting in 57

1106 overrepresented hits identified in melarsoprol survivor populations compared to input

1107 populations.

1108

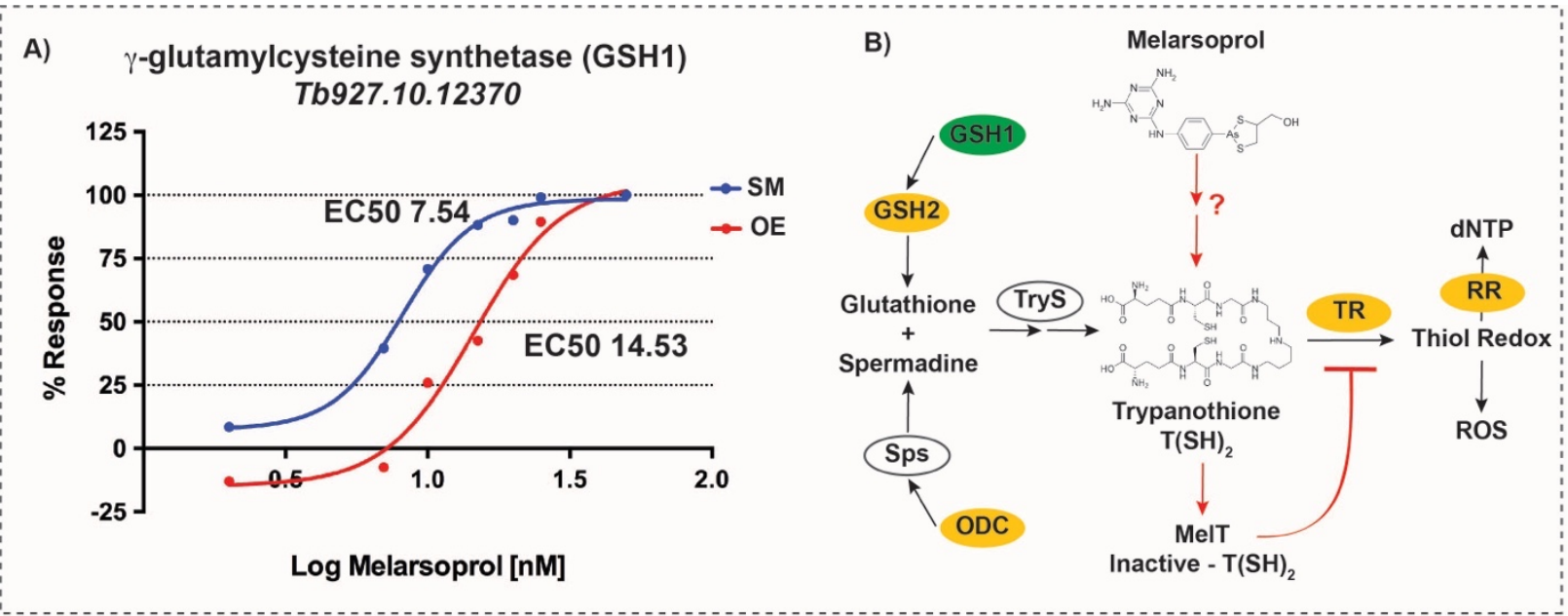

C)

Tb927.7.2780

(Putative Gene Expression)

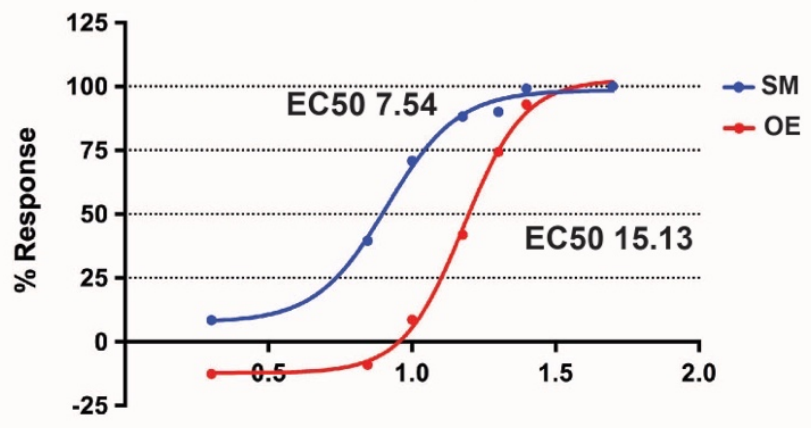

Log Melarsoprol [nM]
D) Tb927.9.15020

(Putative Flagellar Localization)

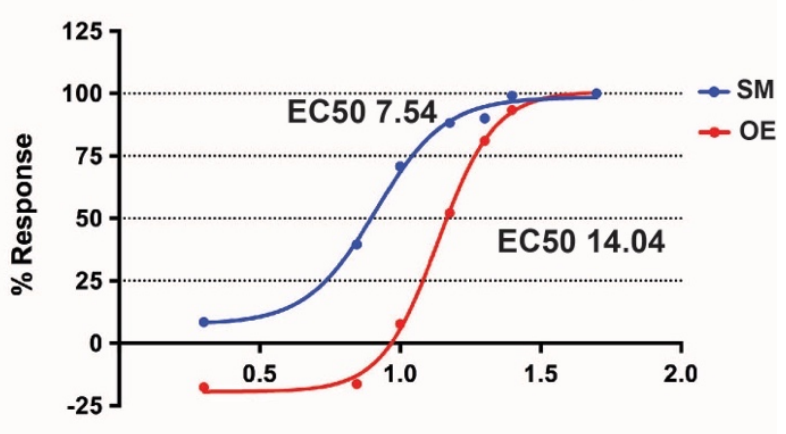

Log Melarsoprol [nM] 
$11127,10,15,20,25$, and $50 \mathrm{nM}$ ) for both parental cell line (SM, blue line) and following Dox

1113 induction of genes identified in the melarsoporol GoF screen (A, C, D red lines labeled

1114 OE). A) Effect of $\gamma$-glutamylcysteine synthetase (Tb927.10.12370, GSH1)

1115 overexpression (red line) on the EC50 of melarsoprol (shown in text on the graph). B)

1116 The associated trypanothione $\left(\mathrm{T}(\mathrm{SH})_{2}\right)$ pathway with $\mathrm{GSH} 1$ is also depicted, in which

1117 the green oval is the overrepresented hit GSH, yellow ovals indicate pathway

1118 components that were modestly effected but did not meet the statistical cutoffs for GoF

1119 hit calling (SUP. 11), and genes encoding enzymes in white ovals were not changed in

1120 the GoF screen. The effects of melarsoprol on $\mathrm{T}(\mathrm{SH})_{2}$ are also depicted as well as the

1121 outcomes of this pathway (panel B). Data panels C) and D) show the effects of

1122 Tb927.7.2780 and Tb927.9.15020 overexpression on the EC50 of melarsoprol,

1123 respectively. All alamarBlue assays were done in biological triplicate for both uninduced

1124 (No Dox, not shown) and induced (+Dox) conditions. Data shown in panel A, C, and D

1125 (red lines) are single experimental representative of all Dox induced biological

1126 replicates. 


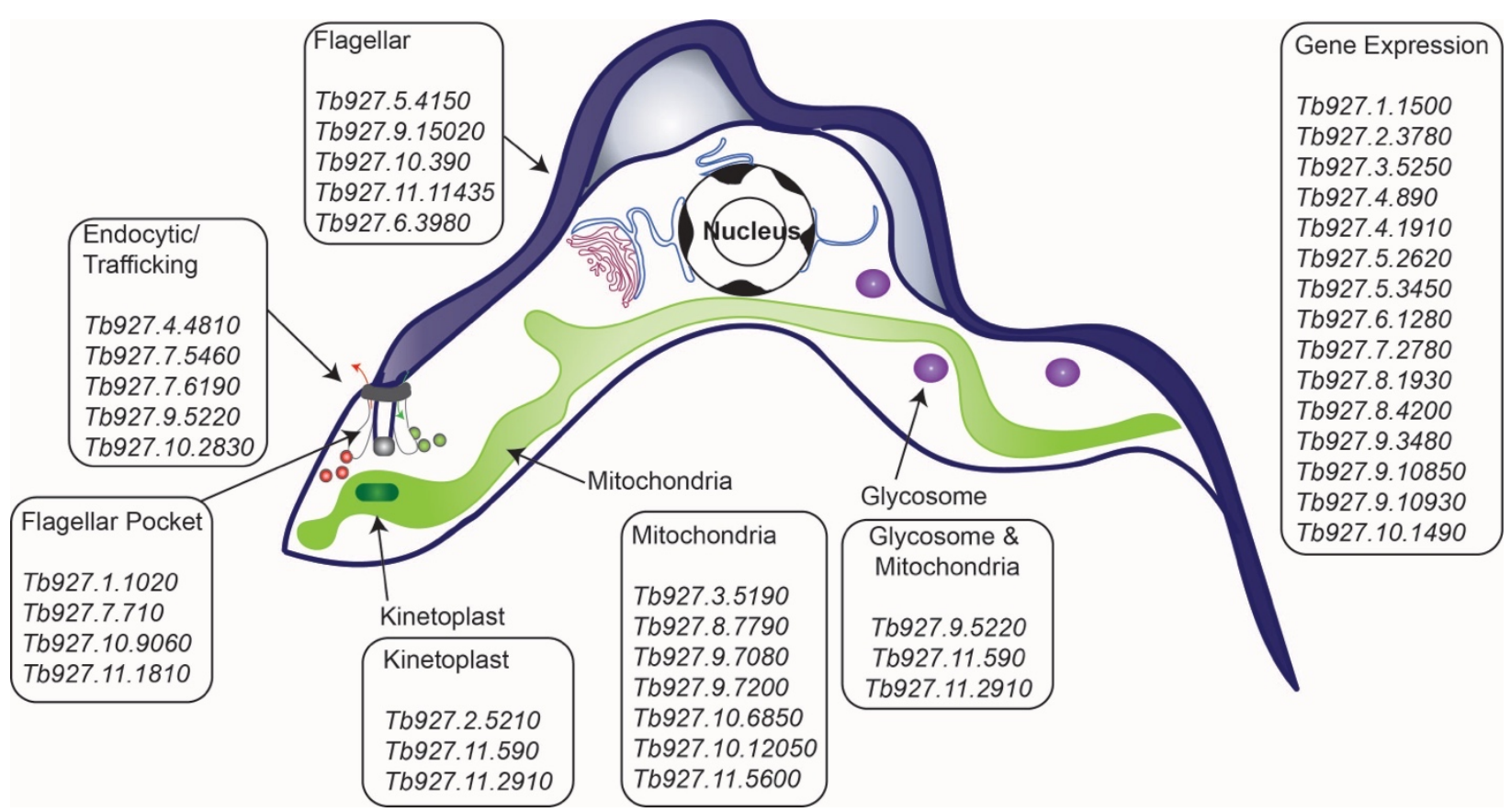

1129 Figure 7. Categories of overrepresented genes in melarsoprol GoF screen

1130 survivor populations. An illustrative diagram of a T. brucei cell is shown with relevant

1131 features highlighted. Boxes of gene numbers indicate major functional or localization

1132 categories for the genes overrepresented in melarsoprol survivor populations. 
Table 1 - ORF eome 384-well oligo plates

\begin{tabular}{|c|c|c|c|}
\hline Plate name & Min length (bp) & Max length (bp) & \# ORFs per plate \\
\hline 0_hypothetical & 102 & 375 & 373 \\
\hline 0_known & 147 & 591 & 315 \\
\hline 1_hypothetical & 375 & 522 & 374 \\
\hline 1_known & 594 & 849 & 365 \\
\hline 2_hypothetical & 522 & 666 & 372 \\
\hline 2_known & 849 & 1056 & 363 \\
\hline 3_hypothetical & 669 & 822 & 382 \\
\hline 3_known & 1056 & 1287 & 367 \\
\hline 4_hypothetical & 822 & 993 & 372 \\
\hline 4_known & 1287 & 1524 & 348 \\
\hline 5_hypothetical & 993 & 1155 & 365 \\
\hline 5_known & 1524 & 1857 & 342 \\
\hline 6_hypothetical & 1158 & 1365 & 375 \\
\hline 6_known & 1857 & 2337 & 362 \\
\hline 7_hypothetical & 1368 & 1635 & 378 \\
\hline 7_known & 2340 & 3504 & 376 \\
\hline 8_hypothetical & 1635 & 1953 & 381 \\
\hline 9_hypothetical & 1953 & 2508 & 381 \\
\hline 10_hypothetical & 2508 & 3501 & 381 \\
\hline hypothetical_last & 3504 & 4488 & 135 \\
\hline known_last & 3507 & 4497 & 138 \\
\hline
\end{tabular}

1134 Table 1. 384-well oligo plates. Plate names, ORF size ranges, and number of oligo

1135 pairs per plate are indicated. 


\begin{tabular}{|c|c|c|c|c|c|}
\hline GENE ID & CATEGORY & DESCRIPTION & LOCALIZATION (Tryptag.org) & $\begin{array}{c}\text { FOLD } \\
\text { CHANGE }\end{array}$ & $\begin{array}{l}\text { P ADJUSTED } \\
\text { DESEQ }\end{array}$ \\
\hline$T b 927.11 .590$ & Mitochondrial* & hypothetical protein, conserved & Strong mitochondrial signal, kinetoplast & 350 & $1.81 \mathrm{E}-39$ \\
\hline$T b 927.10 .12050$ & Mitochondrial* & hypothetical protein, conserved & kinetoplast, mitochondrion & 31 & $2.16 \mathrm{E}-34$ \\
\hline Tb927.9.7200 & Mitochondrial* & hypothetical protein, conserved & kinetoplast, mitochondrion & 31 & $5.61 \mathrm{E}-28$ \\
\hline Tb927.9.7080 & Mitochondrial \& $\mathrm{ER}^{\circ}$ & hypothetical protein, conserved & cytoplasm (patchy, points) & 23 & $1.14 \mathrm{E}-23$ \\
\hline$T b 927.2 .5210$ & Mitochondrial\# & 3-oxoacyl-ACP reductase, putative & mitochondrion, kinetoplast (strong) & 23 & $2.16 \mathrm{E}-34$ \\
\hline$T b 927.11 .2910$ & Mitochondrial* & phosphoglycerate mutase, putative (iPGAM) & mitochondrion $(75 \%)$, kinetoplast $(75 \%)$ & 23 & $6.79 \mathrm{E}-05$ \\
\hline$T b 927.9 .5220$ & Mitochondrialo & conserved protein & endocytic, cytoplasm(weak) & 19 & $2.60 \mathrm{E}-21$ \\
\hline$T b 927.10 .6850$ & Mitochondrial\# & Mitochondrial ribosomal protein S18, putative & cytoplasm(reticulated) & 16 & $3.68 \mathrm{E}-10$ \\
\hline Tb927.8.7790 & Mitochondrialo & zinc finger domain, LSD1 subclass, putative & ND & 13 & $7.88 \mathrm{E}-22$ \\
\hline$T b 927.11 .5600$ & Mitochondrial\# & Archaic Translocase of outer membrane $14 \mathrm{kDa}$ subunit & mitochondrion & 8 & $9.21 \mathrm{E}-11$ \\
\hline$T b 927.3 .5190$ & Mitochondrial+ & hypothetical protein, conserved & mitochondrion & 7 & $8.48 \mathrm{E}-21$ \\
\hline
\end{tabular}

1144 Table 2. Melarsoprol GoF hits with Mitochondrial localization.

Table 3 - Melarsoprol GoF Hits with Flagellar Localiztion

\begin{tabular}{|c|c|c|c|c|c|}
\hline \multirow[b]{2}{*}{ GENE ID } & \multirow[b]{2}{*}{ CATEGORY } & \multirow[b]{2}{*}{ DESCRIPTION } & \multirow[b]{2}{*}{ LOCALIZATION } & \multicolumn{2}{|r|}{ P ADJUSTED } \\
\hline & & & & FOLD CHANGE & DESEQ \\
\hline$T b 927.11 .11435$ & Flagellar+ & dynein light chain lc6 & Strong flagellum axoneme signal & 120 & $1.30 \mathrm{E}-26$ \\
\hline Tb927.9.15020 & Flagellar+ & hypothetical protein, conserved & Weak axoneme signal & 31 & $4.45 \mathrm{E}-20$ \\
\hline Tb927.11.1810 & Flagellar+ & Ring finger domain containing protein, putative & flagellar pocket (ring) & 16 & $1.50 \mathrm{E}-18$ \\
\hline Tb927.10.390 & Flagellar* & hypothetical protein, conserved & Flagellum Matrix Proteome (BSF) & 14 & $2.78 \mathrm{E}-12$ \\
\hline Tb927.5.4150 & Flagellar* & hypothetical protein, conserved & paraflagellar rod & 13 & $6.29 \mathrm{E}-06$ \\
\hline Tb927.7.710 & Flagellar* & heat shock $70 \mathrm{kDa}$ protein, putative (HSP70) & cell tip (anterior), cytoplasm, flagellar cytoplasm & 10 & $3.15 \mathrm{E}-26$ \\
\hline$T b 927.10 .9060$ & Flagellar + & hypothetical protein, conserved & basal body & 8 & $5.86 \mathrm{E}-10$ \\
\hline Tb927.1.1020 & Flagellar + & leucine-rich repeat-containing protein & hook complex & 8 & $1.61 \mathrm{E}-10$ \\
\hline Tb927.6.3980 & Flagellar + & hypothetical protein, conserved & Flagellum, axoneme & 6 & $3.88 \mathrm{E}-10$ \\
\hline
\end{tabular}




\section{SUPPLEMENT LEGENDS}

1158 Supplement 1. Table of oligo pairs from all 21, 384-well plates. The sequences of all

11597245 oligo pairs used to generate the T. brucei ORFeome are included with respect to

1160 their plate "name" and well position in each plate.

1162 Supplement 2. Table of ORFeome amplification tracking. Table indicates the

1163 percent of PCR amplifications that were scored as positive (based on SYBR

1164 assessment) and the number of missing ORFs in reference to each oligo plate. Sub-

1165 tables show the reamplification of "NEG_PICKS" in two addition PCR plates, which

1166 recovered an additional 228 positive PCR products and the final total of 7044 positive

1167 PCR reactions for an overall success rate of $97.2 \%$.

1169 Supplement 3. Map of T. brucei specific pDEST vector, pSUN6. Critical features of 1170 plasmid map are indicated, including: rDNA spacer homology, T7 terminators,

1171 Blasticidin resistance cassette, rRNA promoter, two tetracycline operators, attR site for 1172 pENTR recombination, and the 3' GPEET and 5' Aldolase (long) UTRs.

1174 Supplement 4. Table of all final ORF pools used to make the pENTR ORFeome.

1175 Following the first pENTR_1 and pDEST_1 ORFeome assessments, ORFs missing

1176 from libraries were identified and reselected from original 21 PCR plates to form an

1177 additional 8, size sorted pools. The original 21 pools, 2 pools of PCR reamplifications

1178 (NEG_PICKS) and 8 'missing' ORF pools (1-8_MISS) collectively result in 31 pools for 1179 pENTR cloning. The size ranges and total number of ORFs per pool are indicated. 


\section{Supplement 5. Tables of all missing genes from final pENTR and pDEST}

1182 (pTrypLib) ORFeomes. Consists of four sub-tables for all the ORFs identified as

1183 missing from either pENTR or pDEST by analysis of uniquely or multiply aligned reads:

1184 MISSING_pENTR_UNIQUE, MISSING_pENTR_MULTIPLE,

1185 MISSING_pDEST_UNIQUE, AND MISSING_pDEST_MULTIPLE.

1187 Supplement 6. Assessment of the pTrypLib ORFeome.

1188 A) Histograms showing the distribution of normalized read counts in the pTrypLib

1189 ORFeome in analyses using uniquely aligning reads (left) and allowing multiple

1190 alignments (right). B) The length of each ORF in the pTrypLib Orfeome was plotted

1191 against the normalized read count of the ORF. A best fit line was calculated using linear

1192 regression (shown in white). For uniquely aligned reads this was $y=7.623751$ -

$11930.000673 x$ and for multiply aligned reads this was $y=7.306181-0.000778 x$.

1195 Supplement 7. Sequencing strategy and results from transfected libraries.

1196 A) Library preps from pTrypLib transfected parasites proceed by first fragmenting

1197 genomic DNA, ligating illumina adaptors, and amplifying library introduced ORFs using

1198 a primer complementary to complementary to the attB1 Gateway cloning sequence and

1199 the standard Illumina barcoded reverse primer. Sequencing proceeds using a custom

1200 forward primer complementary to the attB1 Gateway cloning sequence. B) Red

1201 rectangles in the first row represent annotated genes from a section of the T. brucei

1202 chromosome 5. Bars in subsequent rows represent reads that align to the genes in the 
1203 first row. Most reads align to the first 100bp of the gene, as expected from the library

1204 prep and sequencing strategy.

1205

1206 Supplement 8. Assessment of coverage in the GoF INPUT library. A) Histograms

1207 showing the distribution of normalized read counts in the sequencing libraries from

1208 INPUT 1 in analyses using uniquely aligning reads (left) and allowing multiple

1209 alignments (right). B) The length of each ORF in the pTrypLib Orfeome was plotted

1210 against the normalized read count for the INPUT1 sequencing library. A best fit line was

1211 calculated using linear regression (shown in white). For uniquely aligned reads this was

$1212 y=6.046291-0.000423 x$ and for multiply aligned reads this was $y=6.083779-$

$12130.000452 x$.

1214

1215 Supplement 9. Tables of all data pertaining to genes overrepresented in melarsoprol survivor populations.

Supplement 10. Table of categorization and localization of genes overrepresented

1219 in melarsoprol survivor populations.

1220

1221 Supplement 11. Table of trypanothione pathway genes and modest effects

1222 observed in melarsoprol GoF survivor populations.

1223

1224 Supplement 12. Table of melarsoprol GoF hits compared with RIT-seq genetic

1225 screens. 
1227 Supplement 13. Table conservation of the $\mathbf{5 7}$ genes overrepresented in

1228 melarsoprol survivors among other kinetoplastida.

1229

1230 Supplement 14. Table of genes not included in ORFeome design.

1231 